\title{
Degenerations to Unobstructed Fano Stanley-Reisner Schemes
}

\author{
Jan Arthur Christophersen Nathan Owen Ilten
}

\begin{abstract}
We construct degenerations of Mukai varieties and linear sections thereof to special unobstructed Fano Stanley-Reisner schemes corresponding to convex deltahedra. This can be used to find toric degenerations of rank one index one Fano threefolds. In the second part we find many higher dimensional unobstructed Fano and Calabi-Yau Stanley-Reisner schemes. The main result is that the Stanley-Reisner ring of the boundary complex of the dual polytope of the associahedron has trivial $T^{2}$.
\end{abstract}

\section{Introduction}

In [Muk88], Mukai showed that rank one index one Fano threefolds of genus $g \leq 10$ appear as complete intersections in (weighted) projective spaces and homogeneous spaces.

\begin{tabular}{|l|l|l|l|}
\hline Name & Degree & Genus & Embedding \\
\hline$V_{2}^{\prime}$ & 2 & 2 & Sextic in $\mathbb{P}(1,1,1,1,3)$ \\
$V_{4}^{\prime}$ & 4 & 3 & Quartic in $\mathbb{P}^{4}$ \\
$V_{6}$ & 6 & 4 & Intersection of quadric and cubic in $\mathbb{P}^{5}$ \\
$V_{8}$ & 8 & 5 & Intersection of three quadrics in $\mathbb{P}^{6}$ \\
$V_{10}$ & 10 & 6 & Codim. 2 linear subspace of $M_{6}:=Q_{2} \cap G(2,5)$ \\
$V_{12}$ & 12 & 7 & Codim. 7 linear subspace of $M_{7}:=S O(5,10)$ \\
$V_{14}$ & 14 & 8 & Codim. 5 linear subspace of $M_{8}:=G(2,6)$ \\
$V_{16}$ & 16 & 9 & Codim. 3 linear subspace of $M_{9}:=L G(3,6)$ \\
$V_{18}$ & 18 & 10 & Codim. 2 linear subspace of $M_{10}:=\mathbb{G}_{2}$ \\
\hline
\end{tabular}

Here $Q_{2}$ is a generic quadric. The varieties $M_{g}$ are called Mukai varieties. The homogeneous spaces involved are the Grassmannians $G(2,5)$ and $G(2,6)$ associated to $\mathrm{SL}_{5} \mathbb{C}$ and $\mathrm{SL}_{6} \mathbb{C}$, the (even) orthogonal Grassmannian or spinor variety $S O(5,10)$ associated to $\operatorname{Spin}_{10} \mathbb{C}$, the Lagrangian Grassmannian $L G(3,6)$ associated to $\mathrm{Sp}_{6} \mathbb{C}$, and $\mathbb{G}_{2}$ which is associated to the adjoint representation of the exceptional semi-simple Lie Group $G_{2}$. Note that the $V_{2 g-2}$ denote deformation classes as in the original classification of Iskovskih in [Isk78, see Corollary 2.5]below.

In the first part of this paper we compare this series with a special series of Fano StanleyReisner schemes. If $T$ is a combinatorial sphere then the Stanley-Reisner scheme of the join of $T$ and a simplex is Fano (Proposition 2.1). In Section 3 we describe a series of triangulated 2 -spheres $T_{n}, 4 \leq n \leq 11$, with $n$ vertices such that the Stanley-Reisner scheme of the cone over $T_{n}$ is a natural flat degeneration of $V_{2 n-4}$. In fact for $6 \leq g \leq 10$ the Stanley-Reisner scheme of the join of $T_{g+1}$ and a suitable simplex is a degeneration of $M_{g}$.

The series of $T_{n}$ is special for several reasons. To begin with, starting with $T_{4}$, which is the boundary complex of the tetrahedron, $T_{n}$ is gotten from $T_{n-1}$ by starring a vertex into an edge and there is a well defined rule for which edge to star in. This gives a systematic way of generating the Stanley-Reisner degenerations of the $V_{2 g-2}$.

Secondly, for $4 \leq n \leq 10$, the $T_{n}$ are the boundary complexes of the convex deltahedra, i.e. we see all convex deltahedra except the icosahedron. Recall that a deltahedron is a 3-dimensional polytope with regular triangles as faces. There are exactly 8 convex deltahedra as proven in [FvdW47]. Drawings, names and descriptions may be found for example in [Cro97, Figure 2.18]. 
This seems at the moment to be just a nice coincidence but there might be deeper explanation. Although the $T_{n}$ come in a series there is (as usual) no system relating their automorphism groups $\operatorname{Aut}\left(T_{n}\right)$. Yet one can check case by case that for $6 \leq g \leq 9$, if $r_{g}$ is the index of the Fano homogeneous space in the Mukai list, then $\left|\operatorname{Aut}\left(T_{g+1}\right)\right|=24-2 r_{g}$.

Finally, for $4 \leq n \leq 10$, the Stanley-Reisner scheme of the cone over $T_{n}$ is unobstructed. In fact the Stanley-Reisner ring of $T_{n}$ has trivial $T^{2}$. Thus $V_{4}^{\prime}, V_{6}, \ldots, V_{16}$ all degenerate to Fano Stanley-Reisner schemes which are smooth points in the relevant Hilbert schemes. This can be used to find toric varieties to which the Fano threefolds degenerate (Proposition 2.3). The point is that, if a Stanley-Reisner scheme to which we degenerate corresponds to a smooth point in some Hilbert scheme, any toric variety also degenerating to this Stanley-Reisner scheme must deform to a variety corresponding to a general point on the same component of the Hilbert scheme. Our original motivation for this article was in fact to find such toric degenerations, which have become of interest in connection with mirror symmetry, see for example [Prz09] and ILP11.

These results point towards at least two continuations. One can ask if degenerations to Stanley-Reisner schemes help find toric degenerations of other Fano threefolds. This is the subject of a separate paper [CI] where we, for $d \leq 12$, study the Hilbert scheme of degree $d$ smooth Fano threefolds in their anticanonical embeddings. We use this to classify all possible degenerations of these varieties to canonical Gorenstein toric Fanos.

Taking another direction, one could ask for higher dimensional combinatorial spheres with trivial $T^{2}$. This is the subject of the second part of this paper. It is based on the observation that our $T_{9}$ is boundary complex of the triaugmented triangular prism, which again is the dual polytope of the 2-dimensional associahedron. Let $\mathcal{A}_{n}$ be the boundary complex of the dual of the $(n-4)$-dimensional associahedron and $A_{n}$ its Stanley-Reisner ring. The main result of the second part of this paper is Theorem 5.3 which states that $T_{A_{n}}^{2}=0$ for all $n$. For the sake of completeness we also compute $T_{A_{n}}^{1}$ and describe the versal deformation of $\operatorname{Proj}\left(A_{n} \otimes_{k} k\left[x_{0}, \ldots, x_{m}\right]\right)$.

The $T_{n}$ in dimension two appear as edge starrings and unstarrings of $\mathcal{A}_{6}$. In the last section we generalize this and use edge starrings and unstarrings of $\mathcal{A}_{n}$ to find many more combinatorial spheres with trivial $T^{2}$. Our Corollary 6.4 shows that if $r_{1}, \ldots, r_{m}$ are integers with $n>r_{i} \geq 4$ and

$$
\sum_{i=1}^{m} r_{i}=n+3(m-1)
$$

then $\mathcal{A}_{n}$ is as a stellar subdivision of $\mathcal{A}_{r_{1}} * \mathcal{A}_{r_{2}} * \cdots * A_{r_{m}}$ via (different) series of edge starrings. This yields many intermediate $(n-4)$-spheres whose Stanley-Reisner ring has trivial $T^{2}$, generalizing the sequence $T_{6}, \ldots, T_{9}$.

In dimension 2 there is exactly one edge starring of $\mathcal{A}_{6}$ yielding a sphere with $T^{2}=0$, namely $T_{10}$ and any edge starring of $T_{10}$ has non-trivial $T^{2}$. We finish this paper by listing all 74 combinatorial 3 -spheres with trivial $T^{2}$ coming from successive edge starrings of $\mathcal{A}_{7}$.

Several results needed to prove $T_{A_{n}}^{2}=0$ are valid in general for flag complexes and we include them in a separate Section 4. To ensure that general linear sections $G(2, n)$ correspond to generic points on Hilbert scheme components (needed for Corollary 5.4) we prove some results on deformations of complete intersections in rigid Fano varieties which may be of general interest, Proposition 2.4 and Corollary 2.5 ,

Acknowledgments. We are grateful to Kristian Ranestad for helpful discussions. Much of this work was done while the second author was visiting the University of Oslo funded by "småforskmidler". 


\section{Preliminaries}

\subsection{Simplicial complexes and Stanley-Reisner Schemes}

We now recall some basic facts about simplicial complexes and Stanley-Reisner schemes, see for example [Sta96]. Let $[n]$ be the set $\{0, \ldots, n\}$ and $\Delta_{n}$ be the full simplex $2^{[n]}$. An abstract simplicial complex is any subset $\mathcal{K} \subset \Delta_{n}$ such that if $f \in \mathcal{K}$ and $g \subset f$, then $g \in \mathcal{K}$. Elements $f \in \mathcal{K}$ are called faces; the dimension of a face $f$ is $\operatorname{dim} f:=\# f-1$. Zero-dimensional faces are called vertices and we denote the set of vertices by $V(\mathcal{K})$. One-dimensional faces are called edges. By $\Delta_{-1}$ we will denote the simplicial complex consisting solely of the empty set. Two simplicial complexes are isomorphic if there is a bijection of the vertices inducing a bijection of all faces. We will not differentiate between isomorphic complexes.

Given two simplicial complexes $\mathcal{K}$ and $\mathcal{L}$, their join is the simplicial complex

$$
\mathcal{K} * \mathcal{L}=\{f \vee g \mid f \in \mathcal{K}, g \in \mathcal{L}\} .
$$

If $f \in \mathcal{K}$ is a face, we may define

- the link of $f$ in $\mathcal{K} ; \operatorname{lk}(f, \mathcal{K}):=\{g \in \mathcal{K}: g \cap f=\emptyset$ and $g \cup f \in \mathcal{K}\}$,

- the open star of $f$ in $\mathcal{K} ; \operatorname{st}(f, \mathcal{K}):=\{g \in \mathcal{K}: f \subseteq g\}$, and

- the closed star of $f$ in $\mathcal{K} ; \overline{\operatorname{st}}(f, \mathcal{K}):=\{g \in \mathcal{K}: g \cup f \in \mathcal{K}\}$.

Notice that the closed star is the subcomplex $\overline{\operatorname{st}}(f, \mathcal{K})=\bar{f} * \operatorname{lk}(f, \mathcal{K})$. If $f$ is an $r$-dimensional face of $\mathcal{K}$, define the valency of $f, \nu(f)$, to be the number of $(r+1)$-dimensional faces containing $f$. Thus $\nu(f)$ equals the number of vertices in $\operatorname{lk}(f, \mathcal{K})$.

The geometric realization of $\mathcal{K}$, denoted $|\mathcal{K}|$, is defined as

$$
|\mathcal{K}|=\left\{\alpha:[n] \rightarrow[0,1] \mid\{i \mid \alpha(i) \neq 0\} \in \mathcal{K} \text { and } \sum_{i} \alpha(i)=1\right\} .
$$

In this paper we will be interested in the cases where $\mathcal{K}$ is a combinatorial sphere or ball. A combinatorial $n$-sphere is a simplicial complex for which $|\mathcal{K}|$ is $P L$-homeomorphic to the boundary of $\Delta_{n+1}$. A combinatorial $n$-ball is a simplicial complex for which $|\mathcal{K}|$ is $P L$-homeomorphic to $\Delta_{n}$. In general a simplicial complex $\mathcal{K}$ is a combinatorial $n$-manifold (with boundary) if for all non-empty faces $f \in \mathcal{K},|\operatorname{lk}(f, \mathcal{K})|$ is a combinatorial sphere (or ball) of dimension $n-\operatorname{dim} f-1$.

If $b \subseteq V(\mathcal{K})$, denote by $\bar{b}$ the full simplex which is the power set of $b$ and $\partial b=\bar{b} \backslash\{b\}$ its boundary. We recall the notion of stellar exchange defined in Pac91. (See also Vir93.) Assume $\mathcal{K}$ is a complex with a non-empty face $a$ such that $\operatorname{lk}(a, \mathcal{K})=\partial b * L$ for some non-empty set $b$ and $b$ is not a face of $\operatorname{lk}(a, \mathcal{K})$. We can now make a new complex $\mathrm{Fl}_{a, b}(\mathcal{K})$ by removing $\overline{\operatorname{st}}(a)=\partial b * \bar{a} * L$ and replacing it with $\partial a * \bar{b} * L$,

$$
\mathrm{Fl}_{a, b}(\mathcal{K}):=(\mathcal{K} \backslash(\partial b * \bar{a} * L)) \cup \partial a * \bar{b} * L .
$$

If $|b|=1$, that is if $b$ is a new vertex $v$, then the procedure $\mathrm{Fl}_{f, v}(\mathcal{K})$ is classically known as starring $v$ at the face $f$ and we denote the result as $\operatorname{star}(f, \mathcal{K}$ ). (If $f$ also is a vertex we are just renaming $f$ with $v$.) A complex $\mathcal{K}^{\prime}$ is known as a stellar subdivision of $\mathcal{K}$ if there exists a series $\mathcal{K}=\mathcal{K}_{0}, \mathcal{K}_{1}, \ldots, \mathcal{K}_{r}=\mathcal{K}^{\prime}$ such that $\mathcal{K}_{i}=\operatorname{star}\left(f, \mathcal{K}_{i-1}\right)$ for some face $f \in K_{i-1}$.

To any simplicial complex $\mathcal{K} \subset \Delta_{n}$, we associate a square-free monomial ideal $I_{\mathcal{K}} \subset$ $\mathbb{C}\left[x_{0}, \ldots, x_{n}\right]$

$$
I_{\mathcal{K}}:=\left\langle x_{p} \mid p \in \Delta_{n} \backslash \mathcal{K}\right\rangle
$$

where for $p \in \Delta_{n}, x_{p}:=\prod_{i \in p} x_{i}$. This gives rise to the Stanley-Reisner ring $A_{\mathcal{K}}:=\mathbb{C}\left[x_{0}, \ldots, x_{n}\right] / I_{\mathcal{K}}$ and a corresponding projective scheme $\mathbb{P}(\mathcal{K}):=\operatorname{Proj} A_{\mathcal{K}}$ which we call a Stanley-Reisner scheme. The scheme $X:=\mathbb{P}(\mathcal{K})$ "looks" like the complex $\mathcal{K}$ : each face $f \in \mathcal{K}$ corresponds to some $\mathbb{P}^{\operatorname{dim} f} \subset X$ and the intersection relations among these projective spaces are identical to those 
of the faces of $\mathcal{K}$. In particular, facets of $\mathcal{K}$ correspond to the irreducible components of $X$. If $\mathcal{K}$ is pure dimensional then the degree of $\mathbb{P}(\mathcal{K})$ will be the number of facets of $\mathcal{K}$. We also have

$$
H^{p}\left(\mathbb{P}(\mathcal{K}), \mathcal{O}_{\mathbb{P}(\mathcal{K})}\right) \simeq H^{p}(\mathcal{K} ; \mathbb{C}),
$$

by a result of Hochster, see $\mathrm{AC10}$, Theorem 2.2].

We also can make the affine scheme $\mathbb{A}(\mathcal{K})=\operatorname{Spec} A_{\mathcal{K}}$. If $f$ is a subset of $V(\mathcal{K})$, let $D_{+}\left(x_{f}\right) \subseteq$ $\mathbb{P}(\mathcal{K})$ be the chart corresponding to homogeneous localization of $A_{\mathcal{K}}$ by the powers of $x_{f}$. Then $D_{+}\left(x_{f}\right)$ is empty unless $f \in \mathcal{K}$ and if $f \in \mathcal{K}$ then

$$
D_{+}\left(x_{f}\right)=\mathbb{A}(\operatorname{lk}(f, \mathcal{K})) \times\left(k^{*}\right)^{\operatorname{dim} f} .
$$

If $\mathcal{K}$ is an orientable combinatorial manifold without boundary then the canonical sheaf is trivial ([BE91, Theorem 6.1]). Thus a smoothing of such a $\mathbb{P}(\mathcal{K})$ would yield smooth schemes with trivial canonical bundle and structure sheaf cohomology equaling $H^{p}(\mathcal{K} ; \mathbb{C})$. In particular if $\mathcal{K}$ is a combinatorial sphere then a smoothing of $\mathbb{P}(\mathcal{K})$, if such exists, is Calabi-Yau. We shall see that certain balls correspond in this way to Fano schemes.

The combinatorial nature of Stanley-Reisner schemes also makes their deformation theory more accessible than usual and has been studied in [AC04 and AC10. We will apply results from these papers throughout.

\subsection{Cotangent cohomology of Stanley-Reisner schemes}

We recall one of the descriptions in [AC04] of the multi-graded pieces of $T_{A_{\mathcal{K}}}^{i}$ for any simplicial complex $\mathcal{K}$. We refer also to this paper, [AC10] and the references therein to standard works for definitions of the various cotangent cohomology spaces.

We recall first some geometric constructions on simplicial complexes. To every non-empty $f \in \mathcal{K}$, one assigns the relatively open simplex $\langle f\rangle \subseteq|\mathcal{K}|$;

$$
\langle f\rangle=\{\alpha \in|\mathcal{K}| \mid \alpha(i) \neq 0 \text { if and only if } i \in f\} .
$$

On the other hand, each subset $Y \subseteq \mathcal{K}$, i.e. $Y$ is not necessarily a subcomplex, determines a topological space

$$
\langle Y\rangle:= \begin{cases}\bigcup_{f \in Y}\langle f\rangle & \text { if } \emptyset \notin Y \\ \operatorname{cone}\left(\bigcup_{f \in Y}\langle f\rangle\right) & \text { if } \emptyset \in Y .\end{cases}
$$

In particular, $\langle\mathcal{K} \backslash\{\emptyset\}\rangle=|\mathcal{K}|$ and $\langle\mathcal{K}\rangle=|\operatorname{cone}(\mathcal{K})|$ where cone $(\mathcal{K})$ is the simplicial complex $\Delta_{0} * \mathcal{K}$.

Define

$$
\begin{gathered}
U_{b}=U_{b}(\mathcal{K}):=\{f \in \mathcal{K}: f \cup b \notin \mathcal{K}\} \\
\widetilde{U}_{b}=\widetilde{U}_{b}(\mathcal{K}):=\{f \in \mathcal{K}:(f \cup b) \backslash\{v\} \notin \mathcal{K} \text { for some } v \in b\} \subseteq U_{b} .
\end{gathered}
$$

Notice that $U_{b}=\widetilde{U}_{b}=\mathcal{K}$ unless $\partial b$ is a subcomplex of $\mathcal{K}$. If $b \notin \mathcal{K}$ and $\partial b \subseteq \mathcal{K}$ or $b \in \mathcal{K}$, define

$$
L_{b}=L_{b}(\mathcal{K}):=\bigcap_{b^{\prime} \subset b} 1 \mathrm{k}\left(b^{\prime}, \mathcal{K}\right) .
$$

We have

$$
\mathcal{K} \backslash U_{b}=\left\{\begin{array}{ll}
\emptyset \\
\overline{\operatorname{st}}(b)
\end{array} \quad \text { and } \mathcal{K} \backslash \widetilde{U}_{b}= \begin{cases}\partial b * L_{b} & \text { if } b \text { is a non-face } \\
\left(\partial b * L_{b}\right) \cup \overline{\operatorname{st}}(b) & \text { if } b \text { is a face }\end{cases}\right.
$$


Theorem 1.1. ([AC04, Theorem 13]) The homogeneous pieces in degree $\mathbf{c}=\mathbf{a}-\mathbf{b} \in \mathbb{Z}^{|V|}$ (with disjoint supports $a$ and $b)$ of the cotangent cohomology of the Stanley-Reisner ring $A_{\mathcal{K}}$ vanish unless $a \in \mathcal{K}, \mathbf{b} \in\{0,1\}^{|V|}, b \subseteq V(\operatorname{lk}(a, \mathcal{K}))$ and $b \neq \emptyset$. If these conditions are satisfied, we have isomorphisms

$$
T_{A_{\mathcal{K}}, \mathbf{c}}^{i} \simeq H^{i-1}\left(\left\langle U_{b}(\operatorname{lk}(a, \mathcal{K}))\right\rangle,\left\langle\widetilde{U}_{b}(\operatorname{lk}(a, \mathcal{K}))\right\rangle, k\right) \text { for } i=1,2
$$

unless $b$ consists of a single vertex. If $b$ consists of only one vertex, then the above formulae become true if we use the reduced cohomology instead.

Since $T_{A_{\mathcal{K}}, \mathbf{c}}^{i}$ depends only on the supports $a$ and $b$ we may denote it $T_{a-b}^{i}(\mathcal{K})$. We will have use for

Proposition 1.2. ([AC04, Proposition 11]) If $b \subseteq V(\operatorname{lk}(a)$ ), then the map $f \mapsto f \backslash$ a induces isomorphisms $T_{a-b}^{i}(\mathcal{K}) \simeq T_{\emptyset-b}^{i}(\operatorname{lk}(a, \mathcal{K}))$ for $i=1,2$.

We include the following for lack of reference.

Proposition 1.3. If $A$ and $B$ are $k$-algebras then there are exact (split) sequences

$$
0 \rightarrow T_{B}^{i} \otimes_{k} A \rightarrow T_{A \otimes_{k} B}^{i} \rightarrow T_{A}^{i} \otimes_{k} B \rightarrow 0
$$

of cotangent modules for all $i$.

Proof. Consider a cocartesian diagram of rings

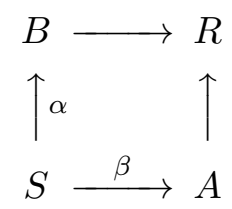

with both $\alpha$ and $\beta$ flat. Then by standard properties of the cotangent modules (see e.g. And74]), if $M$ is a $B$-module, $T^{i}\left(R / A ; M \otimes_{S} A\right) \simeq T^{i}(B / S ; M) \otimes A$. The morphisms $k \rightarrow A \rightarrow A \otimes_{k} B$ yield the Zariski-Jacobi sequence

$$
\cdots \rightarrow T^{i}\left(A \otimes_{k} B / A ; A \otimes_{k} B\right) \rightarrow T_{A \otimes_{k} B}^{i} \rightarrow T^{i}\left(A / k ; A \otimes_{k} B\right) \rightarrow \ldots
$$

Since $B$ is a free $k$-module $T^{i}\left(A / k ; A \otimes_{k} B\right) \simeq T_{A}^{i} \otimes_{k} B$ and the isomorphism above yields $T^{i}\left(A \otimes_{k} B / A ; A \otimes_{k} B\right) \simeq T_{B}^{i} \otimes_{k} A$. Thus the sequence reads

$$
\cdots \rightarrow T_{B}^{i} \otimes_{k} A \rightarrow T_{A \otimes_{k} B}^{i} \rightarrow T_{A}^{i} \otimes_{k} B \rightarrow T_{B}^{i+1} \otimes_{k} A \rightarrow \ldots .
$$

Switching $A$ and $B$ gives a natural section to $T_{A \otimes_{k} B}^{i} \rightarrow T_{A}^{i} \otimes_{k} B$, so the map is surjective and the result follows.

\subsection{Dual associahedra}

By $\mathcal{A}_{n}$ we denote the $n-4$ dimensional simplicial complex which is the boundary complex of the dual polytope of the associahedron. The associahedron (also known as the Stasheff polytope) plays a role in many fields and various generalizations and realizations have appeared in the recent literature, see e.g. the introduction in [HL07] and the references therein. For our purposes the description of $\mathcal{A}_{n}$ given by Lee in [Lee89] is the most useful.

Consider the $n$-gon and index the vertices in cyclical order by $i=1, \ldots, n$. Denote by $\delta_{i j}$ the diagonal between vertex $i$ and vertex $j$. The set of $\frac{1}{2} n(n-3)$ diagonals will be the vertex set of $\mathcal{A}_{n}$, call it $V_{n}$. A set $\left\{\delta_{i_{1} j_{1}}, \ldots, \delta_{i_{r} j_{r}}\right\}$ of $r+1$ diagonals is an $r$-face of $\mathcal{A}_{n}$ if they do not cross, i.e. they partition the $n$-gon into a union of $r+2$ polygons. The facets of $\mathcal{A}_{n}$ correspond 
therefore to the triangulations of the $n$-gon with $n$ vertices. The number of facets is thus the Catalan number

$$
c_{n-2}=\frac{1}{n-2}\left(\begin{array}{c}
2(n-3) \\
n-3
\end{array}\right) .
$$

The automorphism group of $\mathcal{A}_{n}$ is the dihedral group $D_{n}$ and the action is induced by the natural action on the $n$-gon.

For small $n$ we have $\mathcal{A}_{3}=\{\emptyset\}, \mathcal{A}_{4}$ is two vertices with no edge which we denote $S^{0}, \mathcal{A}_{5}$ is the boundary of the pentagon and $\mathcal{A}_{6}$ is the boundary complex of the triaugmented triangular prism.

\section{Degenerations to unobstructed Fano Stanley-Reisner schemes}

We state and prove here general results we will apply to special cases in this paper and in [CI]. Consider a triangulated $n$-ball $B$. Since triangulations of spheres are degenerate Calabi-Yau, one may ask under what conditions the boundary complex corresponds to the anticanonical divisor of $\mathbb{P}(B)$.

Proposition 2.1. Let $T$ be any combinatorial sphere. For $m \geq 0$ consider the variety $X=$ $\mathbb{P}\left(T * \Delta_{m}\right)$. Then $\omega_{X} \cong \mathcal{O}_{X}(-m-1)$. In particular, $X$ is Fano and if $m=0$ the natural embedding is anticanonical.

Proof. Note that $A_{T * \Delta_{m}}=A_{T}\left[x_{0}, \ldots, x_{m}\right]$. The canonical module $\omega_{A_{T}}$ of the Stanley-Reisner ring $A_{T}$ equals $A_{T}$ as graded module, see [Sta96, section 7]. By e.g. [Eis95, 21.11], it follows that $\omega_{A_{T * \Delta_{m}}}=A_{T * \Delta_{m}}(-m-1)$.

We will refer to such simplicial complexes as Fano complexes. In this paper we will be mostly interested in the special situation when $T^{2}$ of the Stanley-Reisner ring of the combinatorial sphere vanishes.

Proposition 2.2. If $\mathcal{K}$ is a combinatorial sphere with $T_{A_{\mathcal{K}}}^{2}=0$, then for the Fano scheme $\mathbb{P}\left(\mathcal{K} * \Delta_{m}\right)$, the obstruction space $T_{\mathbb{P}\left(\mathcal{K}_{*} \Delta_{m}\right) / \mathbb{P}^{m+n}}^{2}$ for the local Hilbert functor vanishes. In particular, $\mathbb{P}\left(\mathcal{K} * \Delta_{m}\right)$ is represented by a smooth point in the corresponding Hilbert scheme.

Proof. Since $A_{\mathcal{K} * \Delta_{m}}$ is just the tensor product over $\mathbb{C}$ of $A_{\mathcal{K}}$ with a polynomial ring, $T_{A_{\mathcal{K} * \Delta_{m}}}^{2}$ vanishes as well, see Proposition 1.3. The claim then follows from [AC10, Proposition 5.4] which states among other things that in this case $T_{\mathbb{P}\left(\mathcal{K}_{*} \Delta_{m}\right) / \mathbb{P}^{m+n}}^{2}$ is the degree 0 part of $T_{A_{\mathcal{K} * \Delta_{m}}}^{2}$.

We now turn our attention to degenerations of smooth Fano varieties to toric varieties. Consider some lattice $M$ and some lattice polytope $\nabla \subset M_{\mathbb{Q}}$ in the associated $\mathbb{Q}$-vector space. By $\mathbb{P}(\nabla)$ we denote the toric variety

$$
\mathbb{P}(\nabla)=\operatorname{Proj} \mathbb{C}\left[S_{\nabla}\right]
$$

where $S_{\nabla}$ is the semigroup in $M \times \mathbb{Z}$ generated by the elements $(u, 1), u \in \nabla \cap M$. By Theorem 8.3 and Corollary 8.9 of [Stu96], square-free initial ideals of the toric ideal of $\mathbb{P}(\nabla)$ are exactly the Stanley-Reisner ideals of unimodular regular triangulations of $\nabla$, see loc. cit. for definitions.

Proposition 2.3. Let $V \subseteq \mathbb{P}^{N}$ be a smooth Fano variety which is the generic point on its component in the Hilbert scheme of $\mathbb{P}^{N}$. Let $\mathcal{K}$ be a combinatorial sphere with $T_{A_{\mathcal{K}}}^{2}=0$ and assume $V$ degenerates to $\mathbb{P}\left(\mathcal{K} * \Delta_{m}\right)$. If $\nabla$ is a lattice polytope having a unimodular regular triangulation of the form $\mathcal{K} * \Delta_{m}$, then $V$ degenerates to $\mathbb{P}(\nabla)$. 
Proof. Now $\mathbb{P}(\nabla)$ degenerates to $\mathbb{P}\left(\mathcal{K} * \Delta_{m}\right)$ and $\mathbb{P}\left(\mathcal{K} * \Delta_{m}\right)$ is represented by a smooth point on the corresponding Hilbert scheme (Proposition 2.2). Since $V$ is represented by a general point on the same component, $\mathbb{P}(\nabla)$ must deform to $V$.

Remark. The toric varieties $\mathbb{P}(\nabla)$ appearing in the above proposition are quite special, since they are unobstructed.

Note that if $V$ is a smooth Fano variety with very ample anticanonical divisor, $V$ is a smooth point on a single irreducible component of the relevant Hilbert scheme, cf. [CI, Proposition 2.1]. A generic point on that component will be a smooth Fano variety, to which we may apply 2.3.

In this paper the smooth Fano varieties that appear are linear sections of rational homogeneous manifolds. Recall that a rational homogeneous manifold is of the form $G / P$ for a complex semi-simple Lie group $G$ and parabolic $P$. Rational homogeneous manifolds are Fano and rigid, i.e. $H^{1}(\Theta)=0$, [Bot57, Theorem VII], and we may use this to show that general sections are generic points on their component in the Hilbert scheme of $\mathbb{P}^{N}$.

For schemes $X \subseteq V$ let $\operatorname{Def}_{X / V}$ be the functor of embedded deformations of $X$ in $V$. The forgetful functor $\operatorname{Def}_{X / V} \rightarrow \operatorname{Def}_{X}$ is smooth if $T_{V}^{1}\left(\mathcal{O}_{X}\right)=0$ and if $V$ is smooth this is $H^{1}\left(X,\left(\Theta_{V}\right)_{\mid X}\right)$.

Proposition 2.4. Let $V$ be a subvariety of $\mathbb{P}^{n}$ such that $H^{1}\left(V, \Theta_{V}\right)=0$ and such that Serre duality holds with dualizing sheaf $\mathcal{O}_{V}(-i)$. Let $X$ be a general complete intersection of $V$ defined by $r$ forms of degree $m_{k}$ on $\mathbb{P}^{N}$ with $\sum_{k=1}^{r} m_{k}<i$. Then $H^{1}\left(X,\left(\Theta_{V}\right)_{\mid X}\right)=0$.

Proof. Let $\mathcal{I}_{X}$ be the ideal sheaf of $X$ in $V$ and consider the exact sequence of sheaves

$$
0 \rightarrow \mathcal{I}_{X} \rightarrow \mathcal{O}_{V} \rightarrow \mathcal{O}_{X} \rightarrow 0 .
$$

After tensoring with $\Theta_{V}$ and passing to the long exact sequence of cohomology, we see that the vanishing of $H^{1}\left(X,\left(\Theta_{V}\right)_{\mid X}\right)$ follows from the vanishing of $H^{1}\left(V, \Theta_{V}\right)$ (which we have by assumption) and the vanishing of $H^{2}\left(V, \mathcal{I}_{X} \otimes \Theta_{V}\right)$. We now show the vanishing of the latter.

Let $\mathcal{F}=\bigoplus_{k=1}^{r} \mathcal{O}_{V}\left(-m_{k}\right)$. Since $X \hookrightarrow V$ is a complete intersection, we have a resolution of $\mathcal{I}_{X}$ by the Koszul complex

$$
0 \longrightarrow \bigwedge^{r} \mathcal{F} \stackrel{d_{r}}{\longrightarrow} \cdots \stackrel{d_{3}}{\longrightarrow} \bigwedge^{2} \mathcal{F} \stackrel{d_{2}}{\longrightarrow} \mathcal{F} \stackrel{d_{1}}{\longrightarrow} \mathcal{I}_{X} \longrightarrow 0
$$

which we can split into short exact sequences

$$
0 \longrightarrow \mathcal{I}_{j} \longrightarrow \bigwedge^{j} \mathcal{F} \longrightarrow \mathcal{I}_{j-1} \longrightarrow 0
$$

with $\mathcal{I}_{0}:=\mathcal{I}_{X}$ and $\mathcal{I}_{j}:=\operatorname{ker} d_{j}$. We show that $H^{p}\left(V, \mathcal{I}_{j} \otimes \Theta_{V}\right)=0$ for $p>1$ by induction on $j$. Indeed, $H^{p}\left(V, \mathcal{I}_{r} \otimes \Theta_{V}\right)=0$ since $\mathcal{I}_{r}=0$. Suppose now that $H^{p}\left(V, \mathcal{I}_{j} \otimes \Theta_{V}\right)=0$ for some $j$ and all $p>1$. Then from the long exact sequence of cohomology, we have

$$
H^{p}\left(V, \mathcal{I}_{j-1} \otimes \Theta_{V}\right) \cong H^{p}\left(V, \bigwedge^{j} \mathcal{F} \otimes \Theta_{V}\right) .
$$

But $\bigwedge^{j} \mathcal{F} \otimes \Theta_{V}$ is a direct sum of vector bundles of the form $\mathcal{O}(-l) \otimes \Theta_{V}$ with $l<i$, and by Serre Duality and Kodaira vanishing, we have

$$
H^{p}\left(V, \mathcal{O}(-l) \otimes \Theta_{V}\right) \cong H^{n-p}\left(V, \mathcal{O}(l-i) \otimes \Omega_{V}\right)=0
$$

where $n$ is the dimension of $V$.

Corollary 2.5. Let $V$ be a rational homogeneous manifold embedded in $\mathbb{P}^{N}$ such that $\omega_{V}=$ $\mathcal{O}_{V}\left(-i_{V}\right)$ where $i_{V}$ is the Fano index of $V$. If $X$ is a smooth complete intersection of $V$ defined by $r$ general forms of degree $m_{k}$ on $\mathbb{P}^{N}$ with $\sum_{k=1}^{r} m_{k}<i_{V}$, then $X$ is Fano and a generic point on its component in the Hilbert scheme of $\mathbb{P}^{N}$.

Proof. From Proposition 2.4 we know that $\operatorname{Def}_{X / V} \rightarrow \operatorname{Def}_{X}$ is smooth. In particular every deformation of $X$ arises from moving the linear section in $V$. A general section is therefore a generic point on the Hilbert scheme component. 

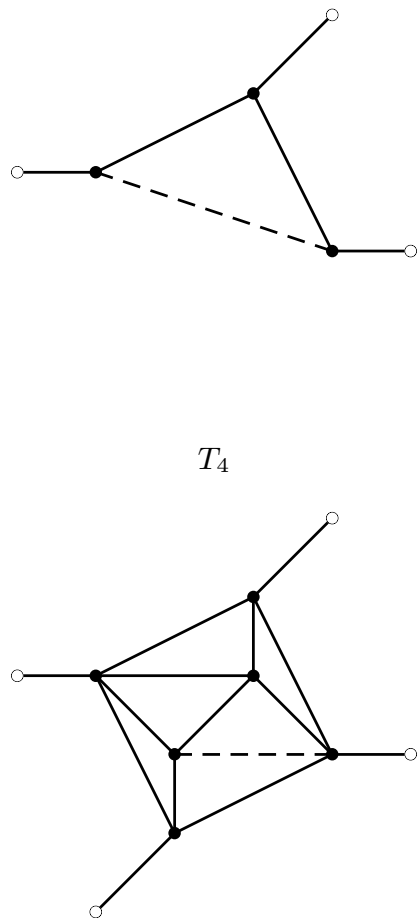

$T_{7}$

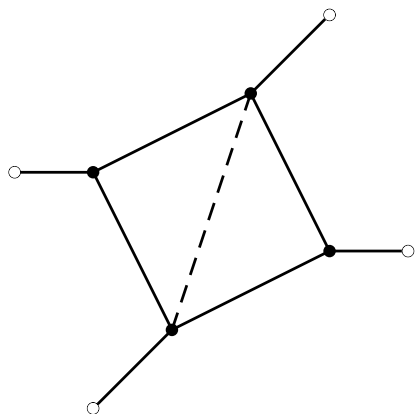

$T_{5}$

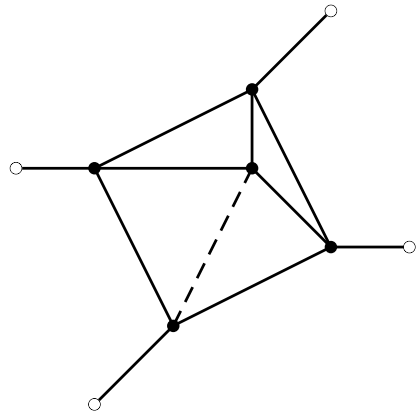

$T_{6}$

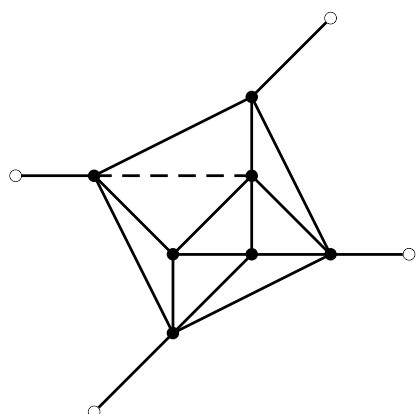

$T_{8}$

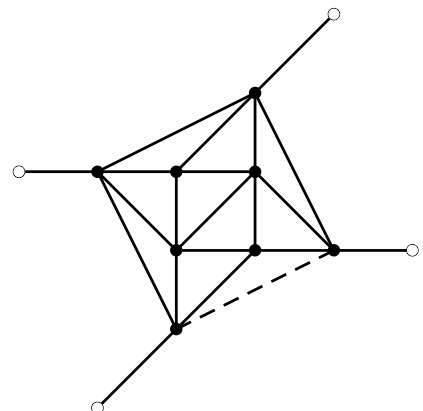

$T_{9}$

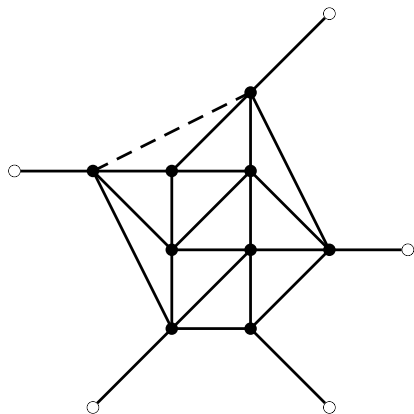

$T_{10}$

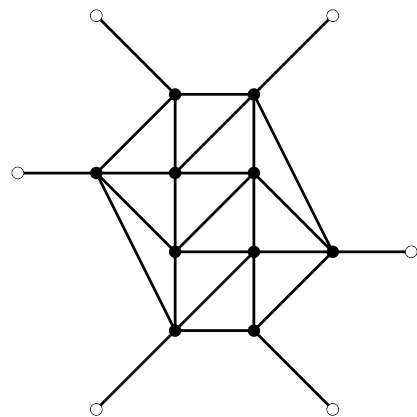

$T_{11}$

Figure 1: Triangulations of the sphere $T_{n}$

\section{Mukai varieties and deltahedra}

We describe a series of triangulated 2-spheres constructed by means of edge starring. Let $T_{4}$ be the boundary complex of the tetrahedron and $T_{5}$ the boundary complex of the triangular bipyramid. For any $6 \leq n \leq 10$, define $T_{n}$ inductively to be $\operatorname{star}\left(f, T_{n-1}\right)$ for any edge $f \in T_{n-1}$ whose link consists of two vertices of valency four. This uniquely determines $T_{n}$.

For $6 \leq n \leq 10$, these are exactly the triangulated spheres where the only vertex valencies are 4 or 5 . The list of $T_{n}, 4 \leq n \leq 10$ coincides with the boundary complexes of the convex deltahedra with 10 or less vertices. Our rule cannot be applied to $T_{10}$, but we define $T_{11}$ to be $\operatorname{star}\left(f, T_{10}\right)$ for any edge $f \in T_{10}$ whose link has one valency-four vertex. These triangulations are pictured in Figure 1 projected from a vertex at infinity. The edges in which we star a vertex are the dashed line segments.

The associahedra appear among the $T_{n}$. We have $T_{9}=\mathcal{A}_{6}$, the octahedron boundary $T_{6}=\mathcal{A}_{4} * \mathcal{A}_{4} * \mathcal{A}_{4}$ and the pentagonal bipyramid boundary $T_{7}=\mathcal{A}_{4} * \mathcal{A}_{5}$.

When $4 \leq n \leq 10$ (the deltahedra case), $T_{n}$ is on the list of triangulated 2-spheres $T$ with 
$T_{A_{T}}^{2}=0$ classified in [IO81, Corollary 2.5]. This may also be proven directly using the results in Section 4. For $\mathcal{K}=T_{11}$, $\underline{\mathrm{AC} 10}$, Theorem 5.6] tells us that $\operatorname{dim} T_{A_{\mathcal{K}, 0}}^{2}=3$.

Theorem 3.1. There is a flat degeneration of $M_{g}$ to $\mathbb{P}\left(T_{g+1} * \Delta_{i_{g}}\right)$ for any $6 \leq g \leq 10$ where $i_{g}$ is one less than the Fano index of $M_{g}$, i.e. $i_{6}=i_{10}=2, i_{7}=7, i_{8}=5$, and $i_{9}=3$.

We wish first to describe the method of proof. Given an ideal $I$ in a polynomial algebra $P$ and a term order $\succ$, let $\operatorname{in}_{\succ}(I)$ be the initial ideal of $I$. There is a flat degeneration of $P / I$ to $P /$ in $_{\succ}(I)$, see e.g. [Eis95, Chapter 15], so we want to find a term order such that $\operatorname{in}_{\succ}\left(I_{M_{g}}\right)$ is the Stanley-Reisner ideal of $T_{g+1} * \Delta_{i_{g}}$.

In [Stu08, Proposition 3.7.4] Sturmfels shows that there is a term order for which the ideal of $G(2, n)$ in the Plücker embedding has initial ideal equal to the Stanley-Reisner ideal of $\mathcal{A}_{n} * \Delta_{n-1}$. He calls this order a circular order and variants of this circular order will be used throughout the proof.

Generators for $I_{M_{g}}$ may be found in the literature, we give references in the proof. Assume we have found a term order such that the ideal generated by the initial terms of these generators is the Stanley-Reisner ideal of $T_{g+1}$. We may then invoke the following useful result of Sturmfels and Zelevinsky.

Let now $I$ be a homogeneous ideal of degree $d$ in $P=\mathbb{C}\left[x_{0}, \ldots, x_{n}\right]$ with $\operatorname{dim} P / I=r+1$ and $\mathcal{G} \subset I$ a finite subset. Suppose the set $\left\{\operatorname{in}_{\succ}(g): g \in \mathcal{G}\right\}$ consists of square-free monomials $x_{q_{1}}, \ldots, x_{q_{s}}, q_{i} \subseteq[n]$.

Proposition 3.2 ([SZ93, Proposition 7.3]). If all minimal (with respect to inclusion) transversal subsets to $\left\{q_{1}, \ldots, q_{s}\right\}$ have the same cardinality $n-r$, and their number is less than or equal to d, then $\mathcal{G}$ is a Gröbner basis with respect to $\succ$.

To rephrase the result recall that a subset $p=\left\{i_{1}, \ldots, i_{k}\right\} \subseteq[n]$ is transversal to $\left\{q_{1}, \ldots, q_{s}\right\}$ if there exists an injective map $f:\{1, \ldots, k\} \rightarrow\{1, \ldots, s\}$ such that $i_{j} \in q_{f(j)}$. Clearly the minimal transversal subsets to $\left\{q_{1}, \ldots, q_{s}\right\}$ are in one to one correspondence with the minimal prime ideals of $\left\langle x_{q_{1}}, \ldots, x_{q_{s}}\right\rangle$. Thus the proposition tells us that if $\left\langle x_{q_{1}}, \ldots, x_{q_{s}}\right\rangle$ is the StanleyReisner ideal of the simplicial complex $\mathcal{K}$ with $n+1$ vertices, $\mathcal{K}$ is pure $r$-dimensional, and the number of facets of $\mathcal{K}$ is less than or equal the degree of $I$, then $\mathcal{G}$ is a Gröbner basis. In particular $\left\langle x_{q_{1}}, \ldots, x_{q_{s}}\right\rangle=\operatorname{in}_{\succ}(I)$.

Proof of Theorem 3.1. Clearly $T_{g+1} * \Delta_{i_{g}}$ is pure dimensional and $\operatorname{dim} T_{g+1} * \Delta_{i_{g}}=\operatorname{dim} M_{g}$. Moreover the degree of $\mathbb{P}\left(T_{g+1} * \Delta_{i_{g}}\right)$ is the number of facets of $T_{g+1}$ which is $2(g-1)$. (For any 2 -sphere the number of facets is 2 (\# vertices -2$)$ by the Euler formula.) This equals the degree of the corresponding Fano 3 -fold in $\mathbb{P}^{g+1}$. Our equations for the Mukai varieties will be in $\mathbb{P}^{g+1+i_{g}}$, so this will also be the degree of the Mukai variety.

By the above remarks it is thus enough to give a set of generators $\mathcal{G}$ and a term order $\succ$ such that $\left\{\operatorname{in}_{\succ}(g): g \in \mathcal{G}\right\}$ are the generators of the Stanley-Reisner ideal of $T_{g+1}$. We do this case by case.

Case $g=6$ and $g=8$ : Grassmannians. We review the argument in Stu08, Proposition 3.7.4] for future reference. Recall that the Grassmannian $G(2, n)$ is defined by the ideal $I$ generated by the $4 \times 4$ Pfaffians of an $n \times n$ antisymmetric matrix with coordinates

$$
\left(\begin{array}{ccccc}
0 & x_{12} & x_{13} & \cdots & x_{1 n} \\
-x_{12} & 0 & x_{23} & \cdots & x_{2 n} \\
-x_{13} & -x_{23} & 0 & \cdots & x_{3 n} \\
\vdots & \vdots & \vdots & \ddots & \vdots \\
-x_{1 n} & -x_{2 n} & -x_{3 n} & \cdots & 0
\end{array}\right)
$$

A circular order $\prec_{\text {circ }}$ is any monomial order which, for $1 \leq i<j<k<l \leq n$, selects the monomial $x_{i k} x_{j l}$ as the lead term in the Pfaffian involving the rows and columns $i, j, k, l$. 
Sturmfels showed that such terms orders exist and that the Pfaffians form a Gröbner basis for them. The initial ideal of $I$ is square-free, and corresponds to $\mathcal{A}_{n} * \Delta_{n-1}$. For $n=6$ this is the simplicial complex $T_{9} * \Delta_{5}$.

When $n=5, \mathcal{A}_{5} * \Delta_{4}=C_{5} * \Delta_{4}$, where $C_{5}$ is the boundary of a pentagon. Now $M_{6}$ is defined by a general quadric in $G(2,5)$. We can degenerate this quadric to $x_{\alpha} x_{\beta}$ where $x_{\alpha}, x_{\beta}$ do not appear in the monomials in the initial ideal of the Pfaffians. The ideal generated by the initial ideal of $I$ and this monomial is the ideal of $T_{7} * \Delta_{2}$.

Case $g=7: S O(5,10)$. Equations for the orthogonal Grassmannian $S O(5,10)$ can be found in [Muk95] (see also [RS00]). Consider the polynomial ring $P$ in the variables $u, x_{i j}$, and $y_{k}$ for $1 \leq i<j \leq 5,1 \leq k \leq 5$. Let $\Phi_{i}(x)$ denote the Pfaffian of the submatrix of (11) for $n=5$ not involving the $i$ th row and column. Then the ideal of $S O(5,10)$ in $\mathbb{P}^{15}$ is given by the five equations of the form

$$
u y_{i}-(-1)^{i} \Phi_{i}(x)
$$

along with the five equations

$$
\left(\begin{array}{ccccc}
0 & x_{12} & x_{13} & x_{14} & x_{15} \\
-x_{12} & 0 & x_{23} & x_{24} & x_{25} \\
-x_{13} & -x_{23} & 0 & x_{34} & x_{35} \\
-x_{14} & -x_{24} & -x_{34} & 0 & x_{45} \\
-x_{15} & -x_{25} & -x_{35} & -x_{45} & 0
\end{array}\right) \cdot\left(\begin{array}{l}
y_{1} \\
y_{2} \\
y_{3} \\
y_{4} \\
y_{5}
\end{array}\right)=0
$$

Consider a circular monomial order on the variables $x_{i j}$ as above, and expand this to any monomial order $\prec$ on $P$ satisfying

$$
u, y_{2}, y_{3}, y_{4} \prec y_{1}, y_{5} \prec x_{i j} \text {. }
$$

Then the initial terms of the above ten equations are generators of the ideal of $\mathbb{P}\left(T_{8} * \Delta_{7}\right)$.

Case $g=9: L G(3,6)$. Equations for the Lagrangian Grassmannian $L G(3,6)$ can be found in [R05]. Consider the polynomial ring $P$ in the variables $u, v, y_{i j}, z_{i j}$ for $1 \leq i \leq j \leq 3$. Let $Y$ and $Z$ be the symmetric matrices

$$
Y=\left(\begin{array}{lll}
y_{11} & y_{12} & y_{13} \\
y_{12} & y_{22} & y_{23} \\
y_{13} & y_{23} & y_{33}
\end{array}\right) \quad Z=\left(\begin{array}{lll}
z_{11} & z_{12} & z_{13} \\
z_{12} & z_{22} & z_{23} \\
z_{13} & z_{23} & z_{33}
\end{array}\right)
$$

and let $M_{i, j}(Y)$ respectively $M_{i, j}(Z)$ denote the $(i, j)$ th minor of $Y$ and $Z$. Then the ideal of $L G(3,6)$ in $\mathbb{P}^{13}$ is given by the 21 equations of the following form:

$$
\begin{array}{rl}
(-1)^{i+j} M_{i, j}(Y)-v z_{i j} & 1 \leq i \leq j \leq 3 \\
(-1)^{i+j} M_{i, j}(Z)-u y_{i j} & 1 \leq i \leq j \leq 3 \\
Y_{i, \cdot} \cdot Z_{\cdot, i}-u v & 1 \leq i \leq 3 \\
Y_{i, \cdot} \cdot Z \cdot, j & 1 \leq i, j \leq 3, \quad i \neq j
\end{array}
$$

Consider now any term order $\prec$ such that

$$
u, v, y_{13}, z_{13} \prec y_{12}, y_{23}, z_{12}, z_{23} \prec y_{i i}, z_{i i} \quad i=1,2,3
$$

and the product of two monomials in the middle group is larger than the product of a monomial from the right with a monomial from the left. These conditions allow for freedom in the four comparisons

$$
y_{i i} z_{i j} ? ? y_{i j} z_{j j} \quad 0 \leq i, j \leq 3, \quad|i-j|=1 .
$$




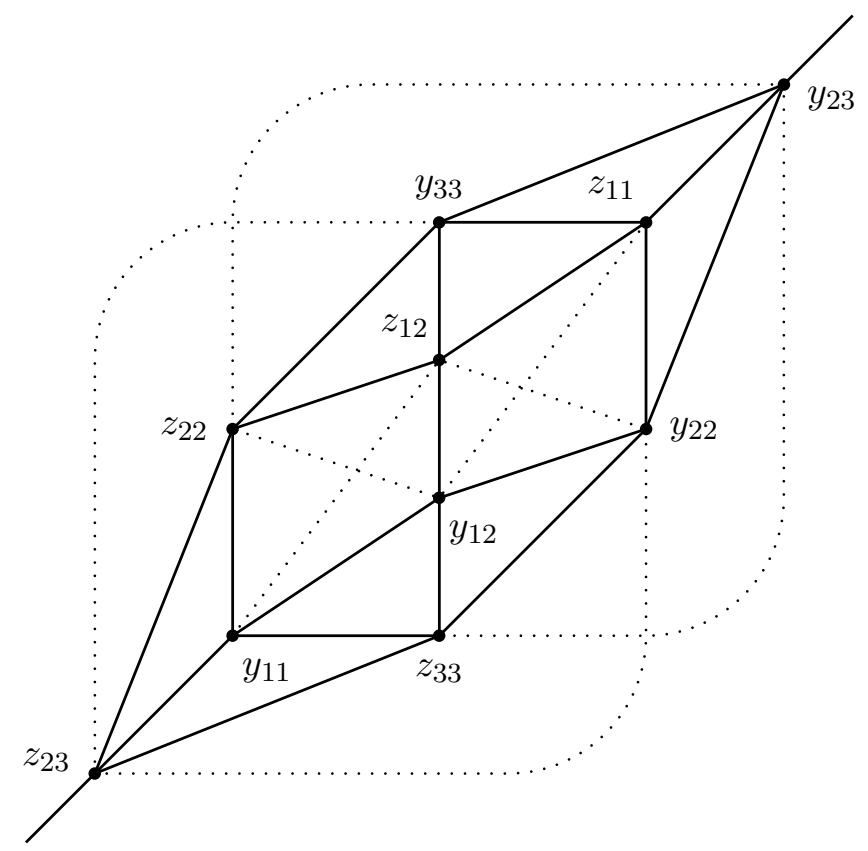

Figure 2: Triangulations coming from initial ideals for $L G(3,6)$

Imposing any further conditions which resolve these four comparisons completely determines the initial terms of the above 21 equations.

In fact, the 16 different possible ideals generated by these terms are all Stanley-Reisner ideals coming from (different) triangulations of the sphere with 10 vertices joined with $\Delta_{3}$; the triangulations can be obtained by always choosing one of the two dotted diagonals in each of the four quadrangles in Figure 2, Exactly two of these triangulations are isomorphic to $T_{10}$. One possible way to get $T_{10}$ is by imposing the additional condition $x_{i i}, y_{i i} \prec x_{j j}, y_{j j}$ for $i<j$.

Case $g=10: \mathbb{G}_{2}$. The $G_{2}$ Grassmannian can be embedded in $G(2,7)$ as a linear section, see for example Kap10]. Let $P$ be the polynomial ring in variables $r, u, w, x_{i j}, y_{i j}, z_{i}$ with $i, j \in\{0,1\}$. Then the ideal of $\mathbb{G}_{2}$ in $\mathbb{P}^{13}$ is generated by the $4 \times 4$ Pfaffians of the matrix

$$
\left(\begin{array}{ccccccc}
0 & -x_{10} & x_{11} & w & y_{11} & y_{10} & u \\
x_{10} & 0 & -v & y_{00} & r & z_{0} & x_{00} \\
-x_{11} & v & 0 & y_{01} & z_{1} & -w-r & x_{01} \\
-w & -y_{00} & -y_{01} & 0 & x_{01} & -x_{00} & v \\
-y_{11} & -r & -z_{1} & -x_{01} & 0 & u & x_{11} \\
-y_{10} & -z_{0} & w+r & x_{00} & -u & 0 & x_{10} \\
-u & -x_{00} & -x_{01} & -v & -x_{11} & -x_{10} & 0
\end{array}\right) .
$$

Note that this is not a minimal generating set for the ideal, it only needs 28 generators.

Consider any term order $\prec$ such that

$$
u, v \prec r, w, x_{i j} \prec y_{i j}, z_{i} \quad i, j \in\{0,1\}
$$

and the product of two monomials in the middle group is larger than the product of a monomial from the right with a monomial from the left. Similar to the $g=9$ case, these conditions allow for freedom in the three comparisons

$$
\begin{aligned}
& x_{00} x_{11} ? ? x_{01} x_{10} \\
& x_{00} y_{01} ? ? x_{01} y_{00} \\
& x_{10} y_{11} ? ? x_{11} y_{10} \text {. }
\end{aligned}
$$

Imposing any further conditions which resolve these three comparisons completely determines the ideals generated by initial terms of the above 35 equations. 


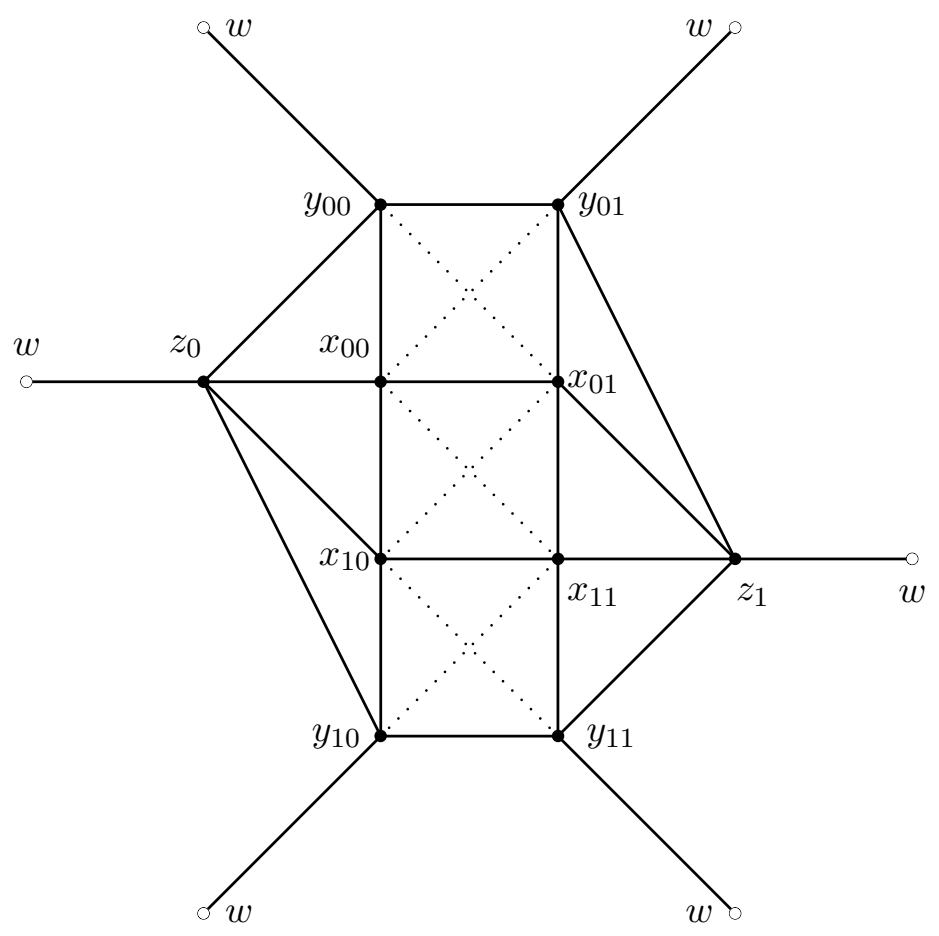

Figure 3: Triangulations coming from initial ideals for $\mathbb{G}_{2}$

The 8 different possible ideals generated by these terms are all Stanley-Reisner ideals coming from (different) triangulations of the sphere with 11 vertices joined with $\Delta_{2}$; the triangulations can be obtained by always choosing one of the two dotted diagonals in each of the three quadrangles in Figure 3. Exactly two of these triangulations are isomorphic to $T_{11}$.

For $-1 \leq k \leq i_{g}-1$ let $h_{0}, \ldots, h_{i_{g}-k-1}$ be general hyperplanes in $\mathbb{P}^{g+1+i_{g}}$. We can degenerate each $h_{j}$ to the coordinate $x_{j}$ corresponding to the $j$ th vertex of $\Delta_{i_{g}}$. Combined with the degeneration of $M_{g}$ in Theorem 3.1, this gives a flat family with general fiber $M_{g} \cap\left\{h_{0}=\cdots=\right.$ $\left.h_{i_{g}-k-1}=0\right\}$ and special fiber $\mathbb{P}\left(T_{g+1} * \Delta_{k}\right)$. We sum this up as

Corollary 3.3. Fix $6 \leq g \leq 10$ and some $-1 \leq k \leq i_{g}-1$. Let $V$ be the intersection of $M_{g}$ with $\left(i_{g}-k\right)$ general hyperplane sections. Then $V$ degenerates to $\mathbb{P}\left(T_{g+1} * \Delta_{k}\right)$.

When $3 \leq g \leq 5$ the $V_{4}^{\prime}, V_{6}$, and $V_{8}$ are complete intersections. Clearly they degenerate to the complete intersections $\mathbb{P}\left(T_{g+1} * \Delta_{0}\right)$. We get therefore Stanley-Reisner degenerations of all rank one index one Fano threefolds of genus $3 \leq g \leq 10$.

Remark. The boundary complex of the icosahedron, the last deltahedron, gives a triangulation of the sphere with 12 vertices such that $T^{2}$ of the corresponding Stanley-Reisner ring vanishes. If we call this complex $T_{12}$, there is no smooth Fano threefold which has an embedded degeneration to $\mathbb{P}\left(T_{12} * \Delta_{0}\right)$. Indeed, the link of the vertex $\Delta_{0}$ corresponds to an affine chart $U_{0}=\operatorname{Spec} A_{T_{12}}$. Since $T_{12}$ has no vertices of valency less then 5 , it follows easily from [AC10, Theorem 4.6] that $U_{0}$ has no deformations in negative degree and is therefore not smoothable. This fits nicely with a "missing" Hilbert scheme component. One computes, e.g. using Theorem 1.1, that $\mathbb{P}\left(T_{12} * \Delta_{0}\right)$ lies on a component of the Hilbert scheme with dimension 174. However, a component of the Hilbert scheme whose general element is a smooth Fano must have dimension 173, 175, 176, or 177 as can be computed from the classification in [MM82 by using [CI, Proposition 2.1].

For $3 \leq g \leq 9$ the above and Corollary 2.5 show that Proposition 2.3 applies so we get 
Corollary 3.4. Let $V$ be a general element in the deformations class $V_{2(g-1)}$ of rank one index one smooth Fano threefolds of genus $3 \leq g \leq 9$. If $\nabla$ is a lattice polytope having a unimodular regular triangulation of the form $T_{g+1} * \Delta_{0}$, then $V$ degenerates to $\mathbb{P}(\nabla)$.

Remark. In the case $g=10$ we know $T_{A_{T_{11}}}^{2} \neq 0$. In fact one may compute that if $\mathcal{K}$ is the Fano complex $T_{11} * \Delta_{0}$ then $T_{\mathbb{P}(\mathcal{K})}^{2}$ is 6 dimensional. The Hilbert scheme locally at this scheme will consist of two components of dimensions 153 and 152. The rank one index one $V_{18}$ is on the 153 dimensional component. This can be used to find toric degenerations of $V_{18}$. Indeed if $\nabla$ is a lattice polytope having a unimodular regular triangulation of the form $T_{11} * \Delta_{0}$ and $h^{0}(\mathbb{P}(\nabla), N)=153$ with $T_{\mathbb{P}(\nabla) / \mathbb{P} 11}^{2}=0$, then $V_{18}$ degenerates to $\mathbb{P}(\nabla)$. These two vector spaces can be computed explicitly via a comparison theorem, see [CI, Proposition 4.2].

\section{Cotangent cohomology for flag complexes}

Recall that $\mathcal{K}$ is called a flag complex if any set of pairwise incident vertices is a face. We may reformulate this as $b \subseteq V(\mathcal{K}), b \notin \mathcal{K}$ and $\partial b \subseteq \mathcal{K}$ implies $|b|=2$. Thus it is clear that $\mathcal{K}$ is a flag complex if and only if $I_{\mathcal{K}}$ is generated by quadratic monomials. For such a quadratic monomial generator $x_{v} x_{w}$ we call the subset $\{v, w\}$ a non-edge. If $\mathcal{K}$ is a flag complex, then so is $\operatorname{lk}(f, \mathcal{K})$ for all $f \in \mathcal{K}$. One simple way to see this is to observe that the Stanley-Reisner ideal of $\operatorname{lk}(f)$ is gotten from $I_{\mathcal{K}}$ by putting $x_{v}=1$ for all $v \in f$.

A flag complex is determined by its edge graph $\Gamma=\Gamma(\mathcal{K})$, since $f \in \mathcal{K}$ if and only if the subgraph of $\Gamma$ induced by the vertices in $f$ is complete. It is the clique complex of its edge graph. On the other hand the clique complex of any simple graph is a flag complex.

When $f \in \mathcal{K}$ we always have $\operatorname{lk}(f, \mathcal{K}) \subseteq L_{f}(\mathcal{K})$, but for a flag complex they are equal. In fact we have

Lemma 4.1. A simplicial complex $\mathcal{K}$ is a flag complex if and only if $\operatorname{lk}(f, \mathcal{K})=L_{f}(\mathcal{K})$ for all faces $f$ with $\operatorname{dim} f \geq 1$.

Proof. Assume first that $\mathcal{K}$ is a flag complex. If $\operatorname{lk}(f, \mathcal{K}) \neq L_{f}$ and $\operatorname{dim} f \geq 1$, there exists a non-empty $g \in \mathcal{K}$ with $g \cup f^{\prime} \in \mathcal{K}$ for all faces $f^{\prime} \subset f$ and $g \cap f^{\prime}=\emptyset$ for all faces $f^{\prime} \subset f$, but $g \notin \operatorname{lk}(f)$. Clearly $f \cap g=\emptyset$, so $f \cup g \notin \mathcal{K}$. If $g^{\prime} \subset g$ then $g^{\prime} \in L_{f}$, so we may choose $g$ minimal, i.e. we may assume $g^{\prime} \cup f \in \mathcal{K}$ for all $g^{\prime} \subset g$. But then, if $b=g \cup f, b \notin \mathcal{K}$ and $\partial b \subseteq \mathcal{K}$. Since $\mathcal{K}$ is a flag complex we must have $|g \cup f|=2$ contradicting $\operatorname{dim} f \geq 1$.

Assume now $\operatorname{lk}(f, \mathcal{K})=L_{f}(\mathcal{K})$ for all faces $f$ with $\operatorname{dim} f \geq 1$. If $|b| \geq 2, b \notin \mathcal{K}$ and $\partial b \subseteq \mathcal{K}$ let $f$ be a facet of $\partial b$ and $v=b \backslash f$. If $f^{\prime} \subset f$ then clearly $v \cap f^{\prime}=\emptyset$. Moreover $f^{\prime} \cup v$ will be in some other facet of $\partial b$, so $v \in L_{f}$. On the other hand $v \cup f=b$ so $v \notin \operatorname{lk}(f)$. Therefore $f$ must be a vertex and $|b|=2$.

Lemma 4.2. If $\mathcal{K}$ is a flag complex and $b \in \mathcal{K}$ and $|b| \geq 2$ then $T_{\emptyset-b}^{i}(\mathcal{K})=0$ for $i=1,2$.

Proof. From Lemma 4.1 we know $\operatorname{lk}(b, \mathcal{K})=L_{b}$. Recall that $\overline{\operatorname{st}}(b)=\bar{b} * \operatorname{lk}(b)$. Thus $\left(\partial b * L_{b}\right) \cup$ $\overline{\mathrm{st}}(b)=(\partial b * \operatorname{lk}(b)) \cup \overline{\mathrm{st}}(b)=\overline{\mathrm{st}}(b)$. It follows that $\widetilde{U}_{b}=U_{b}$ so $T_{\emptyset-b}^{i}(\mathcal{K})=0$ by Theorem 1.1 .

Remark. For $T^{1}$ the above is a rather trivial observation since the ideal is generated by quadrics, but for $T^{2}$ there does not seem to be an easy alternative argument.

Since links of faces in flag complexes are flag complexes, Proposition 1.2 tells us that if we know $T_{\emptyset-b}^{2}(\mathcal{K})$ for flag complexes we know all $T_{a-b}^{2}(\mathcal{K})$.

Proposition 4.3. If $\mathcal{K}$ is a flag complex then $T_{\emptyset-b}^{2}(\mathcal{K})=0$ unless

(i) $b=\{v\}$ is a vertex, then $T_{\emptyset-\{v\}}^{2}(\mathcal{K}) \simeq H^{1}(|\mathcal{K}| \backslash|\overline{\operatorname{st}}(\{v\})|, k)$ or 


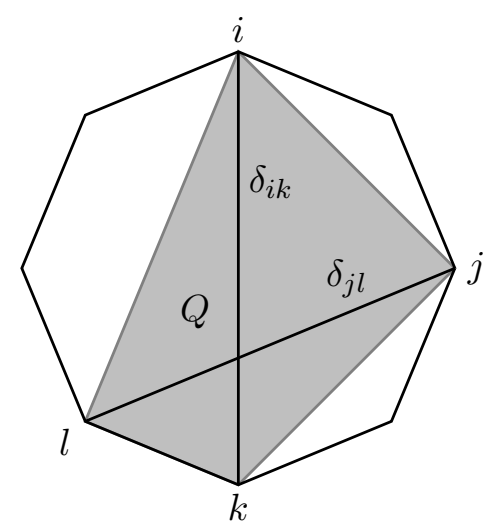

Figure 4: Two crossing diagonals and the quadrangle $Q$.

(ii) $b$ is a non-edge, then $T_{\emptyset-b}^{2}(\mathcal{K}) \simeq \widetilde{H}^{0}\left(|\mathcal{K}| \backslash\left|\partial b * L_{b}\right|, k\right)$.

In particular if $|K|$ is a sphere and $\left|L_{b}\right|$ is contractible then $T_{\emptyset-b}^{2}(\mathcal{K})=0$.

Proof. Since $U_{b}=\widetilde{U}_{b}$ unless $\partial b \subseteq \mathcal{K}$, it follows from Lemma 4.2 and Theorem 1.1 that $T_{\emptyset-b}^{2}(\mathcal{K})=$ 0 unless $b$ is a vertex or non-edge. The isomorphisms are true for all simplicial complexes. By Theorem 1.1 we have $T_{\emptyset-b}^{2} \simeq H^{1}\left(\left\langle U_{b}\right\rangle,\left\langle\widetilde{U}_{b}\right\rangle\right)$. If $b \notin \mathcal{K}$, then $\emptyset \in U_{b}$, so $\left\langle U_{b}\right\rangle$ is a cone and $H^{1}\left(\left\langle U_{b}\right\rangle,\left\langle\widetilde{U}_{b}\right\rangle\right) \simeq \widetilde{H}^{0}\left(\left\langle\widetilde{U}_{b}\right\rangle\right) \simeq \widetilde{H}^{0}\left(|\mathcal{K}| \backslash\left|\partial b * L_{b}\right|, k\right)$. If $b$ is a vertex, then $\widetilde{U}_{b}=\emptyset$.

\section{Cotangent cohomology for the dual associahedron}

We will now apply this to $\mathcal{A}_{n}$. Let $A_{n}$ be the Stanley-Reisner ring of $\mathcal{A}_{n}$. The simplicial complex $\mathcal{A}_{n}$ is a flag complex and the non-edges consist of two crossing diagonals. The Stanley-Reisner ideal of $\mathcal{A}_{n}$ is thus generated by the $\left(\begin{array}{c}n \\ 4\end{array}\right)$ quadratic monomials $x_{i k} x_{j l}$ with $1 \leq i<j<k<l \leq n$ in $k\left[x_{i j}: i<j, \delta_{i j} \in V_{n}\right]$. For a face $f$ let $\mathcal{P}_{f}$ be the set of polygons in the partition of the $n$-gon defined by $f$.

Lemma 5.1. If $f \in \mathcal{A}_{n}$ has dimension $r$ and splits the $n$-gon into $n_{i}$-gons, $i=0, \ldots, r+1$, then $\sum n_{i}=n+2(r+1)$ and $\operatorname{lk}\left(f, \mathcal{A}_{n}\right) \simeq \mathcal{A}_{n_{0}} * \mathcal{A}_{n_{1}} * \cdots * \mathcal{A}_{n_{r+1}}$.

Proof. The facets of $\operatorname{lk}(f)$ may be seen by taking a triangulation of the $n$-gon containing all the diagonals in $f$ and then removing the diagonals in $f$. This clearly gives the splitting.

Let $i, j, k, l$ be labels of vertices on the $n$-gon with $i<j<k<l$. Consider the inscribed quadrangle $Q=Q_{i j k l}$ with vertices $\{i, j, k, l\}$ (see Figure 4). If $i+1 \leq j-1$ then $\delta_{i j}$ is a diagonal splitting the $n$-gon into two polygons. Let $\mathcal{A}_{i j}$ be the dual associahedron corresponding to the polygon with vertices $\{i, i+1, \ldots, j-1, j\}$, i.e. having the common edge $\delta_{i j}$ with $Q$. Finally let $B_{i j}$ be the triangulated ball $B_{i j}=\left\{\delta_{i j}\right\} * \mathcal{A}_{i j} \subseteq \mathcal{A}_{n}$. If $j=i+1$ set $B_{i j}$ to be the empty complex. Now do the same for the other edges of $Q$ to get the 4 pairwise disjoint sub-complexes $B_{i j}, B_{j k}, B_{k l}, B_{l i}$ and set $B_{i j k l}=B_{i j} * B_{j k} * B_{k l} * B_{l i}$.

Lemma 5.2. If $b$ is the non-edge consisting of two crossing diagonals $\delta_{i k}, \delta_{j l}$ with $i<j<k<l$, then $L_{b}\left(\mathcal{A}_{n}\right)=B_{i j k l}$. In particular, if $n \geq 5,\left|L_{b}\right|$ is a $(n-5)$-dimensional ball.

Proof. It is clear that $B_{i j} * B_{j k} * B_{k l} * B_{l i} \subseteq L_{b}$. Assume $f \notin B_{i j} * B_{j k} * B_{k l} * B_{l i}$. Then $f$ must contain a diagonal $\delta$ which is either inside $Q$ or crosses one of the edges of $Q$. In the first case $\delta$ must be either $\delta_{i k}$ or $\delta_{j l}$ and can therefore not be in the corresponding link. In the second case $\delta$ must also cross at least one of $\delta_{i k}, \delta_{j l}$.

The space $\left|L_{b}\right|$ is a ball since the join of two balls is a ball. Note that $\operatorname{dim} B_{i j}=\operatorname{dim} \mathcal{A}_{i j}+1$. The dimension of $L_{b}$ is $\operatorname{dim} B_{i j}+\operatorname{dim} B_{j k}+\operatorname{dim} B_{k l}+\operatorname{dim} B_{l i}+3=n-5$. 
Theorem 5.3. The module $T_{A_{n}}^{2}=0$ for all $n$.

Proof. We will use induction on $n$. For $n=4$ the result is clear since $A_{4}=k[x, y] /(x y)$. We must show that all the spaces $T_{a-b}^{2}\left(\mathcal{A}_{n}\right)$ vanish. If $a \neq \emptyset$ we may use Proposition 1.2, Note that $A_{\mathcal{K} * \mathcal{L}}=A_{\mathcal{K}} \otimes_{k} A_{\mathcal{L}}$. Thus if $T_{A_{k}}^{2}=0$ for all $k<n$, we get $T_{a-b}^{2}\left(\mathcal{A}_{n}\right)=0$ for $a \neq \emptyset$ by Lemma 5.1 and Proposition 1.3. We are left with the case $a=\emptyset$ and this follows directly from Proposition 4.3 and Lemma 5.2 .

In relation to degenerations to toric varieties Proposition 2.3, Corollary 2.5 and the previously referred to [Stu08, Proposition 3.7.4] imply

Corollary 5.4. Let $\nabla$ is a lattice polytope having a unimodular regular triangulation of the form $\mathcal{A}_{n} * \Delta_{m}, 0 \leq m \leq n-1$, then $\mathbb{P}(\nabla)$ is a degeneration of a codimension $n-m-1$ linear section of $G(2, n)$.

Remark. Note that Corollary 5.4 identifies toric degenerations which do not arise via the standard method of finding toric initial ideals of the Plücker ideal. Consider the simple example of

$$
G(2,4)=V\left(x_{12} x_{34}-x_{13} x_{24}+x_{14} x_{23}\right) \subset \mathbb{P}^{5} .
$$

Clearly $G(2,4)$ degenerates to $X=V\left(x_{12} x_{34}-x_{13}^{2}\right)$, but this does not correspond to an initial ideal of $G(2,4)$, as say $V\left(x_{12} x_{34}-x_{13} x_{24}\right)$ does. Nonetheless, we can see this degeneration with our methods: the moment polytope of $X$ has a regular unimodular triangulation of the form $\mathcal{A}_{4} * \Delta_{3}$.

We proceed to compute the $T_{a-b}^{1}\left(\mathcal{A}_{n}\right)$. If $b=\left\{\delta_{i k}, \delta_{j l}\right\}$ consists of two crossing diagonals set $Q_{b}$ to be the corresponding inscribed quadrangle $Q_{i j k l}$.

Theorem 5.5. The structure of $T_{A_{n}}^{1}$ is given by

(i) For $a, b \subseteq V\left(\mathcal{A}_{n}\right)$, if $a \in \mathcal{A}_{n}, b \subseteq V(\operatorname{lk}(a))$ and $b$ consists of two crossing diagonals with $Q_{b} \in \mathcal{P}_{a}$ then $\operatorname{dim}_{k} T_{a-b}^{1}\left(\mathcal{A}_{n}\right)=1$, otherwise $T_{a-b}^{1}\left(\mathcal{A}_{n}\right)=0$.

(ii) There is a one-to-one correspondence between inscribed quadrangles in the $n$-gon and a minimal set of generators for the $A_{n}$-module $T_{A_{n}}^{1}$.

Proof. That $\operatorname{dim} T_{a-b}^{1} \in\{0,1\}$ is a general fact for combinatorial manifolds (without boundary), see ([AC10, Lemma 4.3]). Moreover $T_{a-b}^{1}=0$ if $b$ is a vertex (loc.cit.). Thus by Lemma 4.2, Theorem 1.1 and Lemma 5.2 we are left with the case $b \notin \mathcal{A}_{n}$ and $|b|=2$.

Assume first that $a=\emptyset$. For a combinatorial manifold $\mathcal{K}$, AC10, Theorem 4.6] says $T_{\emptyset-b}^{1}(\mathcal{K}) \neq 0$ iff $\mathcal{K}=L_{b} * \partial b$ i.e. the suspension of $L_{b}$. But if $n \geq 5$, Lemma 5.2 tells us that $\left|L_{b}\right|$ is a ball, so this is impossible unless $n=4$. Indeed we do have $\mathcal{A}_{4}=\{\emptyset\} * \partial b$ where $b$ consists of the two diagonals.

If $a \neq \emptyset$, Proposition 1.2 and Lemma 5.1 tell us that $T_{a-b}^{1} \simeq T_{\emptyset-b}^{1}\left(\mathcal{A}_{n_{0}} * \mathcal{A}_{n_{1}} * \cdots * \mathcal{A}_{n_{r+1}}\right)$ where the product is over the polygons in $\mathcal{P}_{a}=\left\{P_{0}, \ldots, P_{r+1}\right\}$. Now diagonals in different $P_{i}$ will not cross so $b \subseteq V\left(\mathcal{A}_{n_{i}}\right)$ for one $i$ which we may assume is 0 . This means that $L_{b}(\operatorname{lk}(a))=$ $L_{b}\left(\mathcal{A}_{n_{0}}\right) * \mathcal{A}_{n_{1}} * \cdots * \mathcal{A}_{n_{r+1}}$, which is a sphere iff $n_{0}=4$, i.e. $P_{0}=Q_{b}$. On the other hand if $n_{0}=4$ then $\operatorname{lk}(a)=\partial b * \mathcal{A}_{n_{1}} * \cdots * \mathcal{A}_{n_{r+1}}=\partial b * L_{b}$ so $T_{a-b}^{1} \neq 0$. This proves (i).

To prove (ii) we may assume $n>4$. Consider the function that takes an inscribed quadrangle to the set $a(Q)$ consisting of diagonals which are edges of $Q$. Note there could be $1,2,3$ or 4 diagonals in $a(Q)$ depending on the placement of $Q$. If $b(Q)$ is the set of diagonals in $Q$ let $\mathbf{c}(Q)=\chi_{a(Q)}-\chi_{b(Q)} \in \mathbb{Z}^{n(n-3) / 2}$ where $\chi_{A}$ is the characteristic vector of the subset $A$. Then $Q \mapsto$ generator of $T_{\mathbf{c}(Q)}^{1}$ sets up the correspondence.

Indeed from (i) we know that $T_{\mathbf{c}}^{1} \neq 0$ means that $\mathbf{c}=\mathbf{a}-\mathbf{b}$, with disjoint supports $a$ and $b$, and $\mathbf{b}=\chi_{b(Q)}$ for some $Q$ with $Q \in \mathcal{P}_{a}$. The last inclusion implies that $a(Q) \subseteq a$. An element in the one-dimensional $T_{\mathbf{c}}^{1}$ equals $\lambda x^{\mathbf{a}-\chi_{a}(Q)} \cdot$ (generator associated to $Q$ ), where $\lambda$ is some constant and clearly $x^{\mathbf{a}-\chi_{a}(Q)} \neq 0$ in $A_{n}$. 


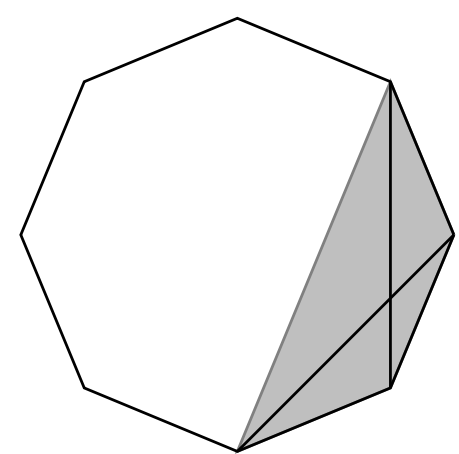

$a$ a vertex

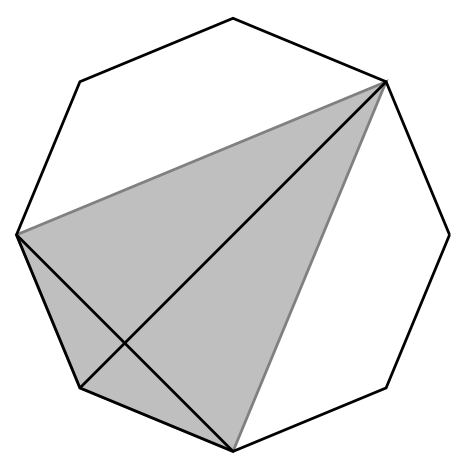

$a$ an edge

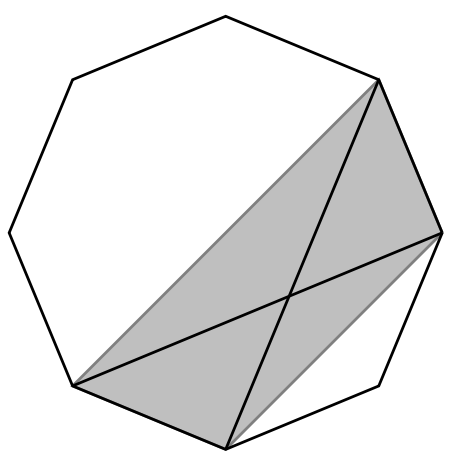

$a$ an edge

Figure 5: Three types of contributions to $T^{1}$.

For the sake of completeness we prove a result about deformation spaces for the StanleyReisner scheme of $\mathcal{A}_{n} * \Delta_{m}$. Let $y_{0}, \ldots, y_{m}$ be the variables corresponding to vertices of $\Delta_{m}$. Since $\operatorname{dim} T_{A_{\mathcal{K}}, \mathbf{c}}^{1}$ is 0 or 1 we may represent a basis element by a rational monomial $x^{\mathbf{c}}$. We are using cyclic indices on the $n$-gon, if e.g. $n=7$ then $j=5, \ldots, 2$ means $j \in\{5,6,7,1,2\}$.

Consider the sets of $T^{1}$ elements

$$
\begin{aligned}
& \left\{\frac{x_{i j} x_{k l}}{x_{i+1, j} x_{i, j-1}}: i=1, \ldots, n, j=i+3, \delta_{k l} \in \operatorname{vert}\left(\operatorname{lk}\left(\left\{\delta_{i j}\right\}\right)\right) \cup\left\{\delta_{i, j}\right\}\right\} \\
& \left\{\frac{x_{i j} y_{k}}{x_{i+1, j} x_{i, j-1}}: i=1, \ldots, n, j=i+3, k=0, \ldots, m\right\} \\
& \left\{\frac{x_{i, j-1} x_{i, j+1}}{x_{i, j} x_{j-1, j+1}}: i=1, \ldots, n, j=i+3, \ldots, i-3\right\} \\
& \left\{\frac{x_{i j} x_{i+1, j-1}}{x_{i+1, j} x_{i, j-1}}: i=1, \ldots, n, j=i+4, \ldots, i-2\right\} .
\end{aligned}
$$

Let $\mathcal{B}_{n, m}$ be the union of these four sets.

Theorem 5.6. If $n \geq 5$ the versal base space of the Stanley-Reisner scheme of $\mathcal{A}_{n} * \Delta_{m}$ is smooth of dimension $\frac{1}{2} n\left(n^{2}-4 n-3\right)+n(m+1)$ unless $n=6$ and $m=-1$. The set $\mathcal{B}_{n, m}$ is a basis for the tangent space. For the exceptional case $\mathcal{A}_{6}$ one must add 1 to the formula because of non-algebraic deformations.

Proof. Let $X$ be the Stanley-Reisner scheme, $A$ the Stanley-Reisner ring and $N=\frac{1}{2} n(n-$ $3)+m$ so that $X \subset \mathbb{P}^{N}$. The local Hilbert functor $\operatorname{Def}_{X / \mathbb{P}^{N}}$ is unobstructed by Theorem 5.3, Proposition 1.3 and [AC10, Proposition 5.4]. The forgetful map $\operatorname{Def}_{X / \mathbb{P}^{N}} \rightarrow \operatorname{Def}_{X}$ is smooth if $H^{1}\left(\Theta_{\mathbb{P}^{N}} \otimes \mathcal{O}_{X}\right)=0$. But by the Euler sequence and a result of Hochster (see e.g. [AC10, Theorem 2.2]) $H^{1}\left(\Theta_{\mathbb{P}^{N}} \otimes \mathcal{O}_{X}\right) \simeq H^{2}\left(\mathcal{O}_{X}\right) \simeq H^{2}\left(\mathcal{A}_{n} * \Delta_{m}, k\right)$. This group vanishes unless $m=-1$ and $n=6$, i.e. the K3 case. This case is covered by Proposition 5.7 below. The dimension of the base space is thus $\operatorname{dim}_{k} T_{X}^{1}$. Since $A$ is Cohen-Macaulay, [Kle79, Theorem 3.9] tells us that $T_{X}^{1} \simeq\left(T_{A}^{1}\right)_{0}$ if $\operatorname{dim} X \geq 3$.

Aside from the special case $\mathcal{A}_{6}$, the $X$ with $\operatorname{dim} X \leq 2$ and $n \geq 5$ correspond to either the boundary of the 5 -gon or the boundary of the 5 -gon $* \Delta_{0}$. In these 2 cases the smoothness of the forgetful map and [AC10, Proposition 5.4] imply that $H^{0}\left(\mathcal{T}_{X}^{1}\right) \simeq\left(T_{A}^{1}\right)_{0}$. On the other hand there is always an exact sequence

$$
0 \rightarrow H^{1}\left(\Theta_{X}\right) \rightarrow T_{X}^{1} \rightarrow H^{0}\left(\mathcal{T}_{X}^{1}\right) \rightarrow H^{2}\left(\Theta_{X}\right) .
$$


A computation in each case shows that $H^{1}\left(\Theta_{X}\right)=H^{2}\left(\Theta_{X}\right)=0$ so we may compute $T_{X}^{1}$ as $\left(T_{A}^{1}\right)_{0}$ directly or as below. For $\mathcal{A}_{6}$ (as for all Stanley-Reisner K3 surfaces, see [AC10, Theorem 5.6]) there is a one dimensional contribution of non-algebraic deformations coming from $H^{1}(\Theta)$.

To compute $\left(T_{A}^{1}\right)_{0}$ we must consider $T_{a-b}^{1}\left(\mathcal{A}_{n}\right)$ where $b$ is a pair of crossing diagonals and $a$ is a vertex or edge. There are three types of contributions as illustrated in Figure 5 , It follows from Theorem 5.5 that if $a$ is a vertex then $T_{a-b}^{1}\left(\mathcal{A}_{n}\right) \neq 0$ iff $a=\left\{\delta_{i j}\right\}$ with $j-i \equiv 3 \bmod n$ and $b=\left\{\delta_{i+1, j}, \delta_{i, j-1}\right\}$. These elements are module generators for the sets (2) and (3) above. The first has $\frac{1}{2} n(n-3)(n-4)$ elements and the second $n(m+1)$ elements. If $a$ is an edge (not containing a vertex as above) then $T_{a-b}^{1}\left(\mathcal{A}_{n}\right) \neq 0$ iff the two diagonals in $a$ are edges of $Q_{b}$ and the other two edges of $Q_{b}$ are edges of the $n$-gon. There are two possibilities leading to the sets (4) of cardinality $n(n-5)$ and (5) of cardinality $\frac{1}{2} n(n-5)$. This adds up to the formula in the statement.

Remark. If $n=4$ then the Stanley-Reisner ring $A$ of $\mathcal{A}_{4} * \Delta_{m}$ is $k\left[x_{0}, x_{1}, y_{0}, \ldots, y_{m}\right] /\left(x_{0} x_{1}\right)$, thus the base space of $\operatorname{Proj} A$ is smooth of dimension $\frac{1}{2}(m+1)(m+2)$.

Even though the base space is smooth it is a non-trivial task to compute the versal family. We give it only in the case $n=6$ as an example. The computations are done by lifting equations and relations using the program Maple. The $D_{6}$ symmetry helps shorten the task. In this case the sets (2) - (5) have cardinality $18,6(m+1), 6$ and 3 . Set

$$
h_{i, i+1}=x_{i-1, i+2}\left(t_{i, 1} x_{i-1, i+2}+t_{i, 2} x_{i-1, i+3}+t_{i, 3} x_{i-2, i+2}+\sum_{k=0}^{m} r_{i, k} y_{k}\right)
$$

for $i=1, \ldots, 6$, where the $t_{i, l}$ and $r_{i, k}$ are parameters. Let $u_{i}$ and $r_{i}$ be parameters dual to the sets (4) and (5). To avoid the non-algebraic deformations in the case $m=-1$ we use the functor $\operatorname{Def}_{X}^{a}$, see [AC10, Section 6].

Proposition 5.7. The versal algebraic family of the Stanley-Reisner scheme of $\mathcal{A}_{6} * \Delta_{m}$ is defined by the 15 equations

$$
\begin{gathered}
x_{i, i+2} x_{i+1, i-1}+h_{i, i+1} x_{i+2, i-1}+s_{i+1} h_{i+1, i+2} h_{i, i-1}-u_{i} h_{i+1, i+2} x_{i+3, i-1} \\
-u_{i+1} h_{i, i-1} x_{i+2, i-2}-u_{i} u_{i+1} h_{i-2, i-1} h_{i+2, i+3}, \quad i=1, \ldots, 6 \\
x_{i, i+3} x_{i+1, i-1}-s_{i} h_{i, i+1} x_{i+3, i-1}-s_{i+1} x_{i+1, i+3} h_{i, i-1}+u_{i} x_{i+1, i+3} x_{i+3, i-1} \\
+u_{i+3}\left(u_{i-1} h_{i, i+1} x_{i, i+2}+u_{i+1} h_{i, i-1} x_{i, i-2}\right)-u_{i+1} s_{i} h_{i, i-1} h_{i+3, i-2}-u_{i+3} s_{i+2} h_{i, i+1} h_{i, i-1} \\
-u_{i-1} s_{i+1} h_{i, i+1} h_{i+2, i+3}-u_{i} u_{i+1} u_{i-1} h_{i+2, i+3} h_{i+3, i-2}, \quad i=1, \ldots, 6 \\
x_{i, i+3} x_{i+2, i-1}+s_{i} x_{i, i+2} x_{i+3, i-1}-s_{i+1} s_{i+2} h_{i, i-1} h_{i+2, i+3}-u_{i} u_{i+2} x_{i+3, i-1}^{2}-u_{i+3} u_{i-1} x_{i, i+2}^{2} \\
+x_{i+3, i-1}\left(u_{i} s_{i+2} h_{i+2, i+3}+u_{i+2} s_{i+1} h_{i, i-1}\right)+x_{i, i+2}\left(u_{i+3} s_{i+2} h_{i, i-1}+u_{i-1} s_{i+1} h_{i+2, i+3}\right) \\
-s_{i} u_{i+1} u_{i-2} h_{i+2, i+3} h_{i, i-1}-u_{i+1} u_{i-2}\left(u_{i+3} u_{i+2} h_{i, i-1}^{2}+u_{i-1} u_{i} h_{i+2, i+3}^{2}\right) \quad i=1, \ldots, 3
\end{gathered}
$$

(all indices are modulo 6 except the $s_{i}$ which must be taken modulo 3 ) over the smooth space with parameters $t_{1,1}, t_{1,2}, t_{1,3}, \ldots, t_{6,1}, t_{6,2}, t_{6,3}, r_{1,0}, \ldots, r_{6, m}, u_{1}, \ldots, u_{6}, s_{1}, s_{2}, s_{3}$.

Remark. The equations are written so that the first 3 terms define linear sections of $G(2,6)$ with the standard Plücker relations. Note this is achieved over the subspace where all $u_{i}=0$. In general one gets easily constructed deformations to sections of $G(2,6)$ when one omits the first order deformations in set (44). On the other hand taking them along complicates matters extremely.

\section{$6 \quad$ Unobstructed spheres via starring and unstarring}

Stellar subdivisions are related to deformations of Stanley-Reisner rings via 
Proposition 6.1. If $\mathcal{K}$ is a simplicial complex and $\mathcal{K}^{\prime}$ is a stellar subdivision of $\mathcal{K}$, then $A_{\mathcal{K}^{\prime}}$ deforms to $A_{\mathcal{K}}$. In particular, if $T_{A_{\mathcal{K}^{\prime}}}^{2}=0$ then $T_{A_{\mathcal{K}}}^{2}=0$.

Proof. We may assume that $\mathcal{K}^{\prime}$ is obtained from starring a simplex $a$ of $X$ at a new vertex $v$, i.e. $\mathcal{K}^{\prime}=(\mathcal{K} \backslash \overline{\operatorname{st}}(a, X)) \cup \partial a * v * \operatorname{lk}(a, \mathcal{K})$. Thus $\operatorname{lk}\left(v, \mathcal{K}^{\prime}\right)=\partial a * L$, where $L=\operatorname{lk}(a, \mathcal{K})$. We get $T_{v-a}^{1}\left(\mathcal{K}^{\prime}\right)=T_{\emptyset-a}^{1}(\partial a * L) \simeq H^{0}(\partial a * L)=k$ by Theorem 1.1.

The corresponding first order deformation with parameter say $t$ is unobstructed since any obstructed $t^{k}$ would have to be in a multigraded part of $T^{2}$ which vanishes since $k \mathbf{a} \notin\{0,1\}^{n}$. Since $\partial a \in \mathcal{K}^{\prime}, x_{a}$ is a generator of the ideal and the deformation is achieved by perturbing this monomial to $x_{a}-t x_{v}$.

We will refer to the opposite procedure of starring in a vertex in a face, i.e. a stellar exchange of the form $\mathrm{Fl}_{v, b}(\mathcal{K})$, as unstarring a vertex. Note that only vertices with special links may be unstarred. We may unstar a vertex $v \in \mathcal{K}$ to form an edge in $\mathcal{K}^{\prime}$ if and only if $\operatorname{lk}(v)=S^{0} * L$ for some subcomplex $L$ and the vertices of the $S^{0}$ must be a non-edge of $\mathcal{K}$. The Stanley-Reisner ideal of $\mathcal{K}^{\prime}$ is gotten from the ideal of $\mathcal{K}$ by removing all monomials containing $x_{v}$ and the monomial $x_{u} x_{v}$. In particular if $\mathcal{K}$ is a flag complex then so is $\mathcal{K}^{\prime}$. We will say the $\mathcal{K}^{\prime}$ is gotten from an edge unstarring.

Let $r, s$ be positive numbers such that $n>r \geq 4$ and $r+s=n+3$. For every such pair we will realize $\mathcal{A}_{n}$ as a stellar subdivision of $\mathcal{A}_{r} * \mathcal{A}_{s}$. This is a generalization of the process yielding $T_{9}=\mathcal{A}_{6}$ from $T_{7}=\mathcal{A}_{5} * \mathcal{A}_{4}$ described in Section 3. In general there will be several different series of unstarrings from $\mathcal{A}_{n}$ to $\mathcal{A}_{r} * \mathcal{A}_{s}$ producing many different combinatorial spheres with unobstructed Stanley-Reisner rings.

Let $\mathcal{A}_{r}$ be the complex of non-crossing diagonals of the polygon with vertices $(1,2, \ldots, r)$ in that cyclic order. Let $\mathcal{A}_{s}$ be the same for the polygon with vertices $(1,2, r, r+1, \ldots, n-1, n)$ in that cyclic order. With this indexing the vertices $\delta_{i j}$ of $\mathcal{A}_{r}$ and $\mathcal{A}_{s}$ are disjoint, but may be interpreted as vertices of $\mathcal{A}_{n}$. The difference in vertex sets is the set

$$
D_{n, r}=\left\{\delta_{i j}: 3 \leq i \leq r-1, r+1 \leq j \leq n\right\}
$$

of $(n-r)(r-3)$ diagonals in $\mathcal{A}_{n}$. On the other hand $\mathcal{A}_{r} * \mathcal{A}_{s}$ contains $(n-r)(r-3)$ edges not in $\mathcal{A}_{n}$, namely the edges in $E_{n, r}=\left\{\left\{\delta_{1, i}, \delta_{2, j}\right\}: 3 \leq i \leq r-1, r+1 \leq j \leq n\right\}$.

Consider the partial order on $D_{n, r}$ given by $\delta_{i j}>\delta_{k l}$ if $j-i>l-k$. Note that $2 \leq j-i \leq n-3$ for $\delta_{i j} \in D_{n, r}$ and that there is a unique maximal element $\delta_{3, n}$ and a unique minimal element $\delta_{r-1, r+1}$. Let $>$ be any total order on $D_{n, r}$ which extends this partial order.

Theorem 6.2. Let $r, s$ be positive numbers such that $n>r \geq 4$ and $r+s=n+3$. Successively unstarring the vertices in the totally ordered set $\left(D_{n, r},>\right)$ by starting with the maximal element and then following the order, realizes $\mathcal{A}_{n}$ as a stellar subdivision of $\mathcal{A}_{r} * \mathcal{A}_{s}$. The sequence of intermediate simplicial complexes yields $(n-r)(r-3)$ triangulated $(n-4)$-spheres whose Stanley-Reisner ring has trivial $T^{2}$.

Proof. By the above remarks it is enough to prove that at each step the chosen $\delta_{i j}$ is unstarrable and that unstarring it adds one of the missing edges in $E_{n, r}$. We do this by induction. Clearly $\operatorname{lk}\left(\delta_{3, n}, \mathcal{A}_{n}\right)=\left\{\left\{\delta_{1,3}\right\},\left\{\delta_{2, n}\right\}\right\} * \mathcal{A}_{n-1}$. We proceed to prove that at each step we may do the unstarring $\mathrm{Fl}_{a, b}$ with $a=\left\{\delta_{i j}\right\}$ and $b=\left\{\delta_{1, i}, \delta_{2, j}\right\}$.

Let $\mathcal{K}$ be the result of unstarring up to $\delta_{i j}$. We must prove that $\operatorname{lk}\left(\delta_{i j}, \mathcal{K}\right)$ is of the form $\left\{\left\{\delta_{1, i}\right\},\left\{\delta_{2, j}\right\}\right\} * L$. This is the same as saying that the only non-edge in $\operatorname{lk}\left(\delta_{i j}, \mathcal{K}\right)$ containing either $\delta_{1, i}$ or $\delta_{2, j}$ is $\left\{\delta_{1, i}, \delta_{2, j}\right\}$. The original link of $\delta_{i j}$ in $\mathcal{A}_{n}$ was $\mathcal{A}_{i+n-j+1} * \mathcal{A}_{j-i+1}$ corresponding to the diagonal splitting the $n$-gon in two. The latter of these has not changed during the previous unstarrings and we may disregard it.

In the former the diagonals crossing $\delta_{1, i}$ were $\delta_{k, l}$ for $2 \leq k \leq i$ and $j \leq l \leq n$. During the unstarring these have been removed except for those with $k=2$, but these have been put into 
edges with $\delta_{1, i}$ except for the case $l=j$. Thus $\left\{\delta_{1, i}, \delta_{2, j}\right\}$ is the only non-edge containing $\delta_{1, i}$. The same argument works for $\delta_{2, j}$ and we have proven the result.

Remark. In fact one can say more, in the notation of the proof, one can prove that

$$
\operatorname{lk}\left(\delta_{i j}, \mathcal{K}\right) \simeq\left\{\left\{\delta_{1, i}\right\},\left\{\delta_{2, j}\right\}\right\} * \mathcal{A}_{i} * \mathcal{A}_{n-j+3} * \mathcal{A}_{j-i+1} .
$$

This allows us to compute the $f$-vector at each step. We give here only a formula for the number of facets. To ease the indexing we set $a_{n}=c_{n-2}$ (the Catalan number) to be the number of facets of $\mathcal{A}_{n}$.

Let $D_{n, r}^{>k}$ be the set of diagonals that have been unstarred before coming to step number $k$ in the process. One unstarring decreases the number of facets by half the number of facets in $\operatorname{lk}\left(\delta_{i j}\right)$. The number of facets of the complex at step $k$ is therefore

$$
a_{n}-\sum_{\delta_{i j} \in D_{n, r}^{>k}} a_{i} a_{n-j+3} a_{j-i+1} .
$$

Applying the formula to different total orders on $D_{n, r}$ shows that different orders will in general yield different intermediate triangulated spheres.

Example 6.3. Clearly one could always chose the order $(i, j) \leq(k, l)$ if $j<l$ or $j=l$ and $i \geq k$.

We may iterate the splitting in Theorem 6.2 to get more unobstructed Stanley-Reisner rings. Note that the final object $\mathcal{A}_{4} * \mathcal{A}_{4} * \cdots * \mathcal{A}_{4}$ is the boundary of the hyper-octahedron, the join of $S^{0}$ with itself $n-3$ times.

Corollary 6.4. If $r_{1}, \ldots, r_{m}$ are integers with $n>r_{i} \geq 4$ and

$$
\sum_{i=1}^{m} r_{i}=n+3(m-1)
$$

then $\mathcal{A}_{n}$ is as a stellar subdivision of $\mathcal{A}_{r_{1}} * \mathcal{A}_{r_{2}} * \cdots * A_{r_{m}}$. In particular $\mathcal{A}_{n}$ and all joins $\mathcal{A}_{r_{1}} * \mathcal{A}_{r_{2}} * \cdots * A_{r_{m}}$ are stellar subdivisions of the boundary complex of the $n-4$ dimensional hyper-octahedron. The subdivisions are done by edge-starrings and yield intermediate $(n-4)$ spheres whose Stanley-Reisner ring has trivial $T^{2}$.

Example 6.5. In dimension 2, i.e. $n=6$ the possible splittings are only $(4,5)$ corresponding to $T_{7}$ (the process in Theorem 6.2 also yields $\left.T_{8}\right)$ and $(4,4,4)$ corresponding to the octahedron $T_{6}$. For $n=7$ the possibilities are $(4,6),(5,5),(4,4,5),(4,4,4,4)$.

In dimension 2 we saw that the unobstructed $T_{10}$ was gotten from $T_{9}=\mathcal{A}_{6}$ by an edge starring. We would like to generalize also this construction to higher dimensions to derive unobstructed triangulated spheres by starring vertices into an edge of $\mathcal{A}_{n}$. If we start with a flag complex $\mathcal{K}$ and star a vertex $v$ in an edge $\{u, w\}$ to get $\mathcal{K}^{\prime}$, then the Stanley-Reisner ideal of $\mathcal{K}^{\prime}$ is gotten from the ideal of $\mathcal{K}$ by adding $x_{u} x_{w}$ and all monomials $x_{v^{\prime}} x_{v}$ where $v^{\prime} \notin \operatorname{lk}(\{u, w\}, \mathcal{K})$. Therefore $\mathcal{K}^{\prime}$ is also a flag complex.

In dimension 3 or higher experimentation shows that there are very many series of edge starrings starting in $\mathcal{A}_{n}$ leading to unobstructed Stanley-Reisner rings and we have not been able to find a suitable presentation of them.

We have found one general series, but there are many others. Consider the series of starrings where we successively star vertices $\varepsilon_{k}, 4 \leq k \leq n-2$, into the edges $\left\{\delta_{1,3}, \delta_{k, n}\right\}$ of $\mathcal{A}_{n}$ and let $\mathcal{C}_{n}$ be the end result. 
Theorem 6.6. The module $T_{A_{\mathcal{C}_{n}}}^{2}=0$ for all $n$. Successively starring vertices into the edges $\left\{\delta_{1,3}, \delta_{k, n}\right\}$ of $\mathcal{A}_{n}$ yields a sequence of $n-5$ triangulated $(n-4)$-spheres whose Stanley-Reisner ring has trivial $T^{2}$.

We omit the long and technical proof which consists of a careful case by case check of what links of vertices and the $L_{b}$ for $\mathcal{C}_{n}$ look like. Instead we illustrate what can happen in higher dimensions by presenting a complete list of 74 combinatorial 3-spheres which appear as successive edges starrings of $\mathcal{A}_{7}$ and have Stanley-Reisner ring with trivial $T^{2}$.

The list was constructed as follows. A necessary condition for a 3 -sphere $\mathcal{K}$ to be a flag complex and have $T^{2}(\mathcal{K})=0$ is that the links of all edges must be 4 -gons or 5 -gons, otherwise $T_{a-b}^{2}(\mathcal{K}) \neq 0$ for an edge $a$ with valency $\geq 6$ and suitable $b$. Thus, from a $\mathcal{K}$ with $T^{2}=0$, to get $\mathcal{K}^{\prime}$ with $T^{2}\left(\mathcal{K}^{\prime}\right)=0$ by edge starring the edge $a$ we star in must satisfy

$$
\text { for all edges } e \in \operatorname{lk}(a, \mathcal{K}), \operatorname{lk}(e, \mathcal{K}) \text { is a 4-gon. }
$$

This is because for such an $e, \operatorname{lk}\left(e, \mathcal{K}^{\prime}\right)=\operatorname{star}(a, \operatorname{lk}(e, \mathcal{K}))$. Call edges satisfying (6) legal edges.

Most of the computations are done in Maple. Assume after successive edge starrings we have found a flag complex 3 -sphere $\mathcal{K}$ with $T^{2}=0$. We may compute the automorphism group as the automorphisms of the edge graph, and this we do with polymake (GJ00]). Finding the legal edges may be done in Maple and we choose one for each orbit of the automorphism group. We compute again in Maple the result of starring in one of these edges and check with polymake if this is isomorphic to a complex we all ready have.

Doing this in a systematic manner we get the list of 743 -spheres in Table 1 below. The "Comes from" column explains which edges in which of the previous complexes one may star to get this sphere. Vertices are the original $\delta_{i j}$ of $\mathcal{A}_{7}$ and new vertices $v_{1}, \ldots, v_{8}$ where the index denotes at which step they appear in the starring process.

To ensure that $T^{2}=0$ we still have to check that $T_{\emptyset-b}^{2}=0$ for all non-edges $b$. It is enough to do this for the "final" ones, i.e. those with no legal edges by Proposition 6.1. These are written with boldface in the tables. We use the identity $T_{\emptyset-b}^{2} \simeq H_{1}\left(L_{b}\right)$ from [AC10, Proposition 4.8] to do this in Maple. In all cases $T_{\emptyset-b}^{2}$ did vanish. Based on this and the dimension 2 case we make the following conjecture.

Conjecture 6.7. If $\mathcal{K}$ is a combinatorial sphere and a flag complex with $\operatorname{lk}(f, \mathcal{K})$ a 4-gon or 5-gon for all codimension 2 faces $f$, then $T_{A_{\mathcal{K}}}^{2}=0$.

In the table, we also include $-\chi(\Theta)$, the number of virtual moduli which a potential smoothing of the Fano fourfold $\mathbb{P}\left(\mathcal{K} * \Delta_{0}\right)$ would have, where $\mathcal{K}$ is a sphere in the table. This is computed by using [AC04, Theorem 12] coupled with [CI, Proposition 2.1]. The Hilbert polynomial of $\mathbb{P}\left(\mathcal{K} * \Delta_{0}\right)$ may be computed from the table. It is a function of the $f$-vector of $\mathcal{K} * \Delta_{0}$ which again by the Dehn-Sommerville equations may be computed from the number of vertices and facets in $\mathcal{K}$. One computes that the Hilbert polynomial of $\mathbb{P}\left(\mathcal{K} * \Delta_{0}\right)$ is

$$
\frac{1}{24} f_{3} t^{4}+\frac{1}{12} f_{3} t^{3}+\left(\frac{1}{2} f_{0}-\frac{1}{24} f_{3}\right) t^{2}+\left(\frac{1}{2} f_{0}-\frac{1}{12} f_{3}\right) t+1
$$

where $f_{0}$ is the number of vertices and $f_{3}$ is the number of facets in the sphere $\mathcal{K}$.

Remark. In light of Corollary 6.4 the above process should be implemented starting with the first unobstructed flag complex, namely the boundary complex of the hyper-octahedron. This will certainly lead to many more 3-dimensional combinatorial spheres having Stanley-Reisner ring with trivial $T^{2}$. 


\begin{tabular}{|c|c|c|c|c|}
\hline Vertices & Name & Facets & Comes from & $-\chi(\Theta)$ \\
\hline \multirow{2}{*}{15} & $\overline{K_{1}}$ & 47 & $\left\{\delta_{13}, \delta_{46}\right\} \in \mathcal{A}_{7}$ & 34 \\
\hline & $\overline{K_{2}}$ & 46 & $\left\{\delta_{13}, \delta_{47}\right\} \in \mathcal{A}_{7}$ & 44 \\
\hline \multirow{7}{*}{16} & $K_{3}$ & 51 & $\left\{\delta_{13}, \delta_{47}\right\} \in K_{1}$ & 38 \\
\hline & $K_{4}$ & 51 & $\begin{array}{l}\left\{\delta_{14}, \delta_{57}\right\} \in K_{1} \\
\left\{\delta_{24}, \delta_{57}\right\} \in K_{2}\end{array}$ & 38 \\
\hline & $\overline{K_{5}}$ & 52 & $\left\{\delta_{16}, \delta_{24}\right\} \in K_{1}$ & 28 \\
\hline & $K_{6}$ & 51 & $\begin{array}{l}\left\{\delta_{16}, \delta_{25}\right\} \in K_{1} \\
\left\{\delta_{16}, \delta_{35}\right\} \in K_{2}\end{array}$ & 36 \\
\hline & $\overline{K_{7}}$ & 52 & $\left\{\delta_{13}, \delta_{57}\right\} \in K_{2}$ & 38 \\
\hline & $K_{8}$ & 51 & $\left\{\delta_{14}, \delta_{57}\right\} \in K_{2}$ & 36 \\
\hline & $\overline{K_{9}}$ & 50 & $\left\{\delta_{16}, \delta_{25}\right\} \in K_{2}$ & 46 \\
\hline \multirow{15}{*}{17} & $K_{10}$ & 55 & $\begin{array}{l}\left\{v_{1}, \delta_{47}\right\} \in K_{3} \\
\left\{v_{1}, \delta_{46}\right\} \in K_{7} \\
\left\{\delta_{13}, \delta_{46}\right\} \in K_{9}\end{array}$ & 40 \\
\hline & $K_{11}$ & 56 & $\left\{\delta_{13}, \delta_{57}\right\} \in K_{3}$ & 32 \\
\hline & $K_{12}$ & 56 & $\begin{array}{l}\left\{\delta_{14}, \delta_{57}\right\} \in K_{3} \\
\left\{\delta_{13}, \delta_{47}\right\} \in K_{4}\end{array}$ & 32 \\
\hline & $K_{13}$ & 56 & $\begin{array}{l}\left\{\delta_{16}, \delta_{24}\right\} \in K_{3} \\
\left\{\delta_{13}, \delta_{47}\right\} \in K_{5}\end{array}$ & 32 \\
\hline & $K_{14}$ & 55 & $\begin{array}{l}\left\{\delta_{16}, \delta_{25}\right\} \in K_{3} \\
\left\{\delta_{13}, \delta_{47}\right\} \in K_{6}\end{array}$ & 40 \\
\hline & $K_{15}$ & 56 & $\begin{array}{l}\left\{\delta_{16}, \delta_{35}\right\} \in K_{3} \\
\left\{\delta_{16}, \delta_{25}\right\} \in K_{5}\end{array}$ & 32 \\
\hline & $K_{16}$ & 55 & $\begin{array}{l}\left\{\delta_{27}, \delta_{36}\right\} \in K_{3} \\
\left\{\delta_{37}, \delta_{46}\right\} \in K_{4}\end{array}$ & 42 \\
\hline & $K_{17}$ & 55 & $\left\{\delta_{37}, \delta_{46}\right\} \in K_{3}$ & 44 \\
\hline & $K_{18}$ & 56 & $\begin{array}{l}\left\{\delta_{16}, \delta_{24}\right\} \in K_{4} \\
\left\{\delta_{14}, \delta_{57}\right\} \in K_{5}\end{array}$ & 34 \\
\hline & $K_{19}$ & 56 & $\begin{array}{l}\left\{\delta_{24}, \delta_{57}\right\} \in K_{4} \\
\left\{\delta_{14}, \delta_{57}\right\} \in K_{7} \\
\left\{\delta_{13}, \delta_{46}\right\} \in K_{8}\end{array}$ & 32 \\
\hline & $K_{20}$ & 55 & $\begin{array}{l}\left\{\delta_{27}, \delta_{36}\right\} \in K_{4} \\
\left\{\delta_{24}, \delta_{57}\right\} \in K_{9}\end{array}$ & 42 \\
\hline & $K_{21}$ & 57 & $\left\{\delta_{16}, \delta_{46}\right\} \in K_{5}$ & 24 \\
\hline & $K_{22}$ & 56 & $\begin{array}{l}\left\{\delta_{16}, \delta_{24}\right\} \in K_{6} \\
\left\{\delta_{16}, \delta_{35}\right\} \in K_{7}\end{array}$ & 32 \\
\hline & $K_{23}$ & 56 & $\left\{\delta_{13}, \delta_{57}\right\} \in K_{8}$ & 32 \\
\hline & $K_{24}$ & 55 & $\begin{array}{l}\left\{\delta_{26}, \delta_{35}\right\} \in K_{8} \\
\left\{\delta_{26}, \delta_{35}\right\} \in K_{9}\end{array}$ & 38 \\
\hline
\end{tabular}

\begin{tabular}{|c|c|c|c|c|}
\hline Vertices & Name & Facets & Comes from & $-\chi(\Theta)$ \\
\hline \multirow{20}{*}{18} & $K_{25}$ & 60 & $\begin{array}{l}\left\{\delta_{14}, \delta_{57}\right\} \in K_{10} \\
\left\{\delta_{27}, \delta_{36}\right\} \in K_{12} \\
\left\{\delta_{14}, \delta_{57}\right\} \in K_{16} \\
\left\{\delta_{27}, \delta_{36}\right\} \in K_{19} \\
\left\{\delta_{13}, \delta_{47}\right\} \in K_{20}\end{array}$ & 36 \\
\hline & $K_{26}$ & 59 & $\begin{array}{l}\left\{\delta_{16}, \delta_{25}\right\} \in K_{10} \\
\left\{v_{1}, \delta_{47}\right\} \in K_{14}\end{array}$ & 42 \\
\hline & $K_{27}$ & 60 & $\begin{array}{l}\left\{\delta_{16}, \delta_{35}\right\} \in K_{10} \\
\left\{v_{1}, \delta_{47}\right\} \in K_{15} \\
\left\{v_{2}, \delta_{24}\right\} \in K_{22}\end{array}$ & 34 \\
\hline & $K_{28}$ & 60 & $\begin{array}{l}\left\{\delta_{37}, \delta_{46}\right\} \in K_{10} \\
\left\{v_{1}, \delta_{37}\right\} \in K_{17}\end{array}$ & 36 \\
\hline & $K_{29}$ & 61 & $\left\{\delta_{14}, \delta_{57}\right\} \in K_{11}$ & 28 \\
\hline & $K_{30}$ & 61 & $\begin{array}{l}\left\{\delta_{16}, \delta_{35}\right\} \in K_{11} \\
\left\{\delta_{13}, \delta_{57}\right\} \in K_{15}\end{array}$ & 28 \\
\hline & $K_{31}$ & 60 & $\begin{array}{l}\left\{\delta_{37}, \delta_{46}\right\} \in K_{11} \\
\left\{\delta_{37}, \delta_{46}\right\} \in K_{12} \\
\left\{\delta_{37}, \delta_{46}\right\} \in K_{16} \\
\left\{\delta_{13}, \delta_{57}\right\} \in K_{17}\end{array}$ & 38 \\
\hline & $K_{32}$ & 61 & $\begin{array}{l}\left\{\delta_{13}, \delta_{57}\right\} \in K_{12} \\
\left\{\delta_{14}, \delta_{57}\right\} \in K_{13} \\
\left\{\delta_{13}, \delta_{47}\right\} \in K_{18}\end{array}$ & 28 \\
\hline & $K_{33}$ & 61 & $\begin{array}{l}\left\{\delta_{24}, \delta_{57}\right\} \in K_{12} \\
\left\{\delta_{13}, \delta_{47}\right\} \in K_{19}\end{array}$ & 28 \\
\hline & $K_{34}$ & 60 & $\begin{array}{l}\left\{\delta_{16}, \delta_{25}\right\} \in K_{13} \\
\left\{\delta_{26}, \delta_{35}\right\} \in K_{15}\end{array}$ & 36 \\
\hline & $K_{35}$ & 60 & $\begin{array}{l}\left\{\delta_{37}, \delta_{46}\right\} \in K_{13} \\
\left\{\delta_{37}, \delta_{46}\right\} \in K_{15} \\
\left\{\delta_{16}, \delta_{24}\right\} \in K_{17}\end{array}$ & 38 \\
\hline & $K_{36}$ & 60 & $\begin{array}{l}\left\{\delta_{16}, \delta_{24}\right\} \in K_{14} \\
\left\{\delta_{13}, \delta_{47}\right\} \in K_{22} \\
\left\{v_{1}, \delta_{57}\right\} \in K_{23}\end{array}$ & 36 \\
\hline & $K_{37}$ & 60 & $\begin{array}{l}\left\{\delta_{16}, \delta_{35}\right\} \in K_{14} \\
\left\{\delta_{37}, \delta_{46}\right\} \in K_{22} \\
\end{array}$ & 36 \\
\hline & $K_{38}$ & 59 & $\begin{array}{l}\left\{\delta_{37}, \delta_{46}\right\} \in K_{14} \\
\left\{\delta_{16}, \delta_{25}\right\} \in K_{17}\end{array}$ & 46 \\
\hline & $K_{39}$ & 60 & $\left\{\delta_{16}, \delta_{25}\right\} \in K_{15}$ & 36 \\
\hline & $K_{40}$ & 60 & $\begin{array}{l}\left\{\delta_{27}, \delta_{36}\right\} \in K_{15} \\
\left\{\delta_{16}, \delta_{35}\right\} \in K_{16} \\
\left\{\delta_{16}, \delta_{25}\right\} \in K_{18}\end{array}$ & 38 \\
\hline & $K_{41}$ & 61 & $\begin{array}{l}\left\{\delta_{16}, \delta_{46}\right\} \in K_{18} \\
\left\{\delta_{14}, \delta_{57}\right\} \in K_{21}\end{array}$ & 30 \\
\hline & $K_{42}$ & 61 & $\left\{\delta_{15}, \delta_{24}\right\} \in K_{22}$ & 26 \\
\hline & $K_{43}$ & 61 & $\left\{\delta_{16}, \delta_{46}\right\} \in K_{22}$ & 28 \\
\hline & $K_{44}$ & 60 & $\begin{array}{l}\left\{\delta_{26}, \delta_{35}\right\} \in K_{23} \\
\left\{\delta_{13}, \delta_{57}\right\} \in K_{24}\end{array}$ & 34 \\
\hline
\end{tabular}

Table 1: 3 -spheres with $T^{2}(\mathcal{K})=0$ generated by edge starrings. 


\begin{tabular}{|c|c|c|c|c|}
\hline Vertices & Name & Facets & Comes from & $-\chi(\Theta)$ \\
\hline \multirow{17}{*}{19} & $K_{45}$ & 65 & $\begin{array}{l}\left\{\delta_{24}, \delta_{57}\right\} \in K_{25} \\
\left\{\delta_{27}, \delta_{36}\right\} \in K_{33}\end{array}$ & 32 \\
\hline & $K_{46}$ & 65 & $\begin{array}{l}\left\{\delta_{37}, \delta_{46}\right\} \in K_{25} \\
\left\{\delta_{14}, \delta_{57}\right\} \in K_{28} \\
\left\{\delta_{27}, \delta_{46}\right\} \in K_{31}\end{array}$ & 32 \\
\hline & $K_{47}$ & 64 & $\begin{array}{l}\left\{\delta_{16}, \delta_{35}\right\} \in K_{26} \\
\left\{\delta_{16}, \delta_{25}\right\} \in K_{28} \\
\left\{v_{1}, \delta_{47}\right\} \in K_{37} \\
\left\{v_{1}, \delta_{37}\right\} \in K_{38}\end{array}$ & 38 \\
\hline & $K_{48}$ & 64 & $\begin{array}{l}\left\{\delta_{16}, \delta_{25}\right\} \in K_{27} \\
\left\{v_{3}, \delta_{35}\right\} \in K_{37} \\
\left\{v_{1}, \delta_{47}\right\} \in K_{39}\end{array}$ & 38 \\
\hline & $K_{49}$ & 64 & $\begin{array}{l}\left\{\delta_{26}, \delta_{35}\right\} \in K_{27} \\
\left\{v_{3}, \delta_{25}\right\} \in K_{34} \\
\left\{v_{3}, \delta_{24}\right\} \in K_{36} \\
\left\{v_{1}, \delta_{57}\right\} \in K_{44}\end{array}$ & 38 \\
\hline & $K_{50}$ & 65 & $\begin{array}{l}\left\{\delta_{37}, \delta_{46}\right\} \in K_{27} \\
\left\{\delta_{16}, \delta_{35}\right\} \in K_{28} \\
\left\{v_{1}, \delta_{37}\right\} \in K_{35}\end{array}$ & 30 \\
\hline & $K_{51}$ & 65 & $\begin{array}{l}\left\{v_{3}, \delta_{14}\right\} \in K_{29} \\
\left\{v_{3}, \delta_{13}\right\} \in K_{32} \\
\left\{\delta_{14}, \delta_{57}\right\} \in K_{34} \\
\left\{\delta_{26}, \delta_{35}\right\} \in K_{40}\end{array}$ & 32 \\
\hline & $K_{52}$ & 66 & $\begin{array}{l}\left\{\delta_{16}, \delta_{35}\right\} \in K_{29} \\
\left\{\delta_{14}, \delta_{57}\right\} \in K_{30}\end{array}$ & 24 \\
\hline & $K_{53}$ & 66 & $\left\{\delta_{24}, \delta_{57}\right\} \in K_{29}$ & 24 \\
\hline & $K_{54}$ & 65 & $\begin{array}{l}\left\{\delta_{37}, \delta_{46}\right\} \in K_{29} \\
\left\{\delta_{37}, \delta_{46}\right\} \in K_{30} \\
\left\{\delta_{14}, \delta_{57}\right\} \in K_{31} \\
\left\{\delta_{37}, \delta_{46}\right\} \in K_{32} \\
\left\{\delta_{14}, \delta_{57}\right\} \in K_{35} \\
\left\{\delta_{37}, \delta_{46}\right\} \in K_{40}\end{array}$ & 34 \\
\hline & $K_{55}$ & 65 & $\begin{array}{l}\left\{\delta_{16}, \delta_{25}\right\} \in K_{30} \\
\left\{\delta_{13}, \delta_{57}\right\} \in K_{39}\end{array}$ & 32 \\
\hline & $K_{56}$ & 65 & $\begin{array}{l}\left\{\delta_{26}, \delta_{35}\right\} \in K_{30} \\
\left\{\delta_{16}, \delta_{35}\right\} \in K_{34}\end{array}$ & 32 \\
\hline & $K_{57}$ & 66 & $\left\{\delta_{16}, \delta_{24}\right\} \in K_{32}$ & 24 \\
\hline & $K_{58}$ & 64 & $\begin{array}{l}\left\{\delta_{37}, \delta_{46}\right\} \in K_{34} \\
\left\{\delta_{16}, \delta_{25}\right\} \in K_{35} \\
\left\{\delta_{26}, \delta_{35}\right\} \in K_{39}\end{array}$ & 42 \\
\hline & $K_{59}$ & 64 & $\begin{array}{l}\left\{\delta_{37}, \delta_{46}\right\} \in K_{36} \\
\left\{\delta_{37}, \delta_{46}\right\} \in K_{37} \\
\left\{\delta_{16}, \delta_{24}\right\} \in K_{38}\end{array}$ & 42 \\
\hline & $K_{60}$ & 65 & $\begin{array}{l}\left\{\delta_{26}, \delta_{35}\right\} \in K_{37} \\
\left\{\delta_{37}, \delta_{46}\right\} \in K_{42}\end{array}$ & 30 \\
\hline & $K_{61}$ & 64 & $\begin{array}{l}\left\{\delta_{27}, \delta_{36}\right\} \in K_{39} \\
\left\{\delta_{16}, \delta_{25}\right\} \in K_{40}\end{array}$ & 42 \\
\hline
\end{tabular}

\begin{tabular}{|c|c|c|c|c|}
\hline Vertices & Name & Facets & Comes from & $-\chi(\Theta)$ \\
\hline \multirow{10}{*}{20} & $K_{62}$ & 68 & $\begin{array}{l}\left\{v_{4}, \delta_{35}\right\} \in K_{47} \\
\left\{v_{4}, \delta_{25}\right\} \in K_{48}\end{array}$ & 40 \\
\hline & $K_{63}$ & 69 & $\begin{array}{l}\left\{\delta_{26}, \delta_{35}\right\} \in K_{47} \\
\left\{v_{3}, \delta_{37}\right\} \in K_{50} \\
\left\{v_{1}, \delta_{47}\right\} \in K_{60}\end{array}$ & 32 \\
\hline & $K_{64}$ & 69 & $\begin{array}{l}\left\{\delta_{37}, \delta_{46}\right\} \in K_{47} \\
\left\{v_{1}, \delta_{37}\right\} \in K_{59}\end{array}$ & 34 \\
\hline & $K_{65}$ & 68 & $\begin{array}{l}\left\{v_{4}, \delta_{25}\right\} \in K_{48} \\
\left\{\delta_{16}, \delta_{25}\right\} \in K_{49} \\
\left\{v_{3}, \delta_{25}\right\} \in K_{58} \\
\left\{v_{3}, \delta_{24}\right\} \in K_{59}\end{array}$ & 44 \\
\hline & $K_{66}$ & 69 & $\begin{array}{l}\left\{\delta_{37}, \delta_{46}\right\} \in K_{48} \\
\left\{\delta_{16}, \delta_{25}\right\} \in K_{50} \\
\left\{v_{1}, \delta_{37}\right\} \in K_{58}\end{array}$ & 34 \\
\hline & $K_{67}$ & 69 & $\begin{array}{l}\left\{\delta_{37}, \delta_{46}\right\} \in K_{51} \\
\left\{v_{3}, \delta_{14}\right\} \in K_{54} \\
\left\{\delta_{37}, \delta_{46}\right\} \in K_{55} \\
\left\{\delta_{14}, \delta_{57}\right\} \in K_{58} \\
\left\{\delta_{26}, \delta_{35}\right\} \in K_{61}\end{array}$ & 38 \\
\hline & $K_{68}$ & 70 & $\begin{array}{l}\left\{\delta_{26}, \delta_{35}\right\} \in K_{52} \\
\left\{\delta_{14}, \delta_{57}\right\} \in K_{56}\end{array}$ & 28 \\
\hline & $K_{69}$ & 70 & $\begin{array}{l}\left\{\delta_{37}, \delta_{46}\right\} \in K_{52} \\
\left\{\delta_{16}, \delta_{35}\right\} \in K_{54} \\
\left\{\delta_{37}, \delta_{46}\right\} \in K_{57}\end{array}$ & 30 \\
\hline & $K_{70}$ & 69 & $\begin{array}{l}\left\{\delta_{26}, \delta_{35}\right\} \in K_{55} \\
\left\{\delta_{16}, \delta_{25}\right\} \in K_{56} \\
\left\{\delta_{16}, \delta_{35}\right\} \in K_{58}\end{array}$ & 38 \\
\hline & $K_{71}$ & 69 & $\left\{v_{3}, \delta_{35}\right\} \in K_{60}$ & 34 \\
\hline \multirow[t]{2}{*}{21} & $K_{72}$ & 73 & $\begin{array}{l}\left\{\delta_{26}, \delta_{35}\right\} \in K_{62} \\
\left\{v_{3}, \delta_{37}\right\} \in K_{64} \\
\left\{v_{4}, \delta_{25}\right\} \in K_{65} \\
\left\{v_{4}, \delta_{25}\right\} \in K_{66}\end{array}$ & 36 \\
\hline & $K_{73}$ & 73 & $\begin{array}{l}\left\{v_{4}, v_{6}\right\} \in K_{63} \\
\left\{v_{3}, \delta_{37}\right\} \in K_{66} \\
\left\{v_{1}, \delta_{47}\right\} \in K_{71}\end{array}$ & 36 \\
\hline 22 & $K_{74}$ & 77 & $\begin{array}{l}\left\{v_{6}, \delta_{26}\right\} \in K_{72} \\
\left\{v_{4}, v_{6}\right\} \in K_{73}\end{array}$ & 38 \\
\hline
\end{tabular}

Table 1: 3 -spheres with $T^{2}(\mathcal{K})=0$ generated by edge starrings. 


\section{References}

[AC04] Klaus Altmann and Jan Arthur Christophersen, Cotangent cohomology of StanleyReisner rings, Manuscripta Math. 115 (2004), no. 3, 361-378.

[AC10] — Deforming Stanley-Reisner schemes, Math. Ann. 348 (2010), 513-537.

[And74] Michel André, Homologie des algèbres commutatives, Springer-Verlag, 1974.

[BE91] Dave Bayer and David Eisenbud, Graph curves, Adv. Math. 86 (1991), no. 1, 1-40.

[Bot57] Raoul Bott, Homogeneous vector bundles, Ann. of Math. (2) 66 (1957), 203-248.

[CI] Jan Arthur Christophersen and Nathan Owen Ilten, Toric degenerations of low degree Fano threefolds, in preparation.

[Cro97] Peter R. Cromwell, Polyhedra, Cambridge University Press, 1997.

[Eis95] David Eisenbud, Commutative algebra, Graduate Texts in Mathematics, vol. 150, Springer-Verlag, 1995.

[FvdW47] H. Freudenthal and B. L. v. d. Waerden, On an assertion of Euclid, Simon Stevin 25 (1947), 115-121.

[GJ00] Ewgenij Gawrilow and Michael Joswig, polymake: a framework for analyzing convex polytopes, Polytopes - Combinatorics and Computation, Birkhäuser, 2000, pp. 4374 .

[HL07] Christophe Hohlweg and Carsten E. M. C. Lange, Realizations of the associahedron and cyclohedron, Discrete Comput. Geom. 37 (2007), no. 4, 517-543.

[ILP11] Nathan Owen Ilten, Jacob Lewis, and Victor Przyjalkowski, Toric degenerations of Fano threefolds giving weak Landau-Ginzburg models, arXiv:1102.4664v2 [math.AG], 2011.

[IO81] Masi-Nori Ishida and Tadao Oda, Torus embeddings and tangent complexes, Tôhoku Math. Journ. 33 (1981), 337-381.

[IR05] Atanas Iliev and Kristian Ranestad, Geometry of the Lagrangian Grassmannian LG(3,6) with applications to Brill-Noether loci, Michigan Math. J. 53 (2005), no. 2, $383-417$.

[Isk78] V. A. Iskovskih, Fano threefolds. II., Izv. Akad. Nauk SSSR Ser. Mat. 42 (1978), no. $3,506-549$.

[Kap10] Michal Kapustka, Relations between equations of Mukai varieties, arXiv:1005.5557v3 [math.AG], 2010.

[Kle79] Jan O. Kleppe, Deformations of graded algebras, Math. Scand. 45 (1979), 205-231.

[Lee89] Carl W. Lee, The associahedron and triangulations of the n-gon, European J. Combin. 10 (1989), no. 6, 551-560.

[MM82] Shigefumi Mori and Shigeru Mukai, Classification of Fano 3-folds with $B_{2} \geq 2$, Manuscripta Math. 36 (1981/82), no. 2, 147-162.

[Muk88] Shigeru Mukai, Curves, K3 surfaces and Fano 3-folds of genus $\leq 10$, Algebraic geometry and commutative algebra, Vol. I, Kinokuniya, Tokyo, 1988, pp. 357-377. 
[Muk95] _ Curves and symmetric spaces. I, Amer. J. Math. 117 (1995), no. 6, 16271644.

[Pac91] Udo Pachner, PL homeomorphic manifolds are equivalent by elementary shellings, Europ. J. Combinatorics 12 (1991), 129-145.

[Prz09] Victor Przyjalkowski, Weak Landau-Ginzburg models for smooth Fano three-folds, arXiv:0902.4668v2 [math.AG], 2009.

[RS00] Kristian Ranestad and Frank-Olaf Schreyer, Varieties of sums of powers, J. Reine Angew. Math. 525 (2000), 147-181.

[Sta96] Richard P. Stanley, Combinatorics and commutative algebra, second ed., Birkhäuser Boston Inc., 1996.

[Stu96] Bernd Sturmfels, Gröbner bases and convex polytopes, University Lecture Series, vol. 8, American Mathematical Society, 1996.

[Stu08] _ Algorithms in invariant theory, second ed., Texts and Monographs in Symbolic Computation, Springer Verlag, 2008.

[SZ93] Bernd Sturmfels and Andrei Zelevinsky, Maximal minors and their leading terms, Adv. Math. 98 (1993), no. 1, 65-112.

[Vir93] Oleg Viro, Lectures on combinatorial presentations of manifolds, Differential geometry and topology (Alghero, 1992), World Sci. Publishing, 1993, pp. 244-264.

Jan Arthur Christophersen

Matematisk INSTitutT

Postboks 1053 BLINDERN

University of OsLo

N-0316 OSLO, NoRWAy

E-mail address: christop@math.uio.no

Nathan Owen Ilten

Department of Mathematics

University OF CALIFORNIA

BERKELEy CA 94720, USA

E-mail address: nilten@math.berkeley.edu 